\title{
Which fraction of the measured cosmic-ray antiprotons might be due to neutralino annihilation in the galactic halo?
}

\author{
A. Bottino, ${ }^{*}$ F. Donato ${ }^{\dagger}$ and N. Fornengo ${ }^{\ddagger}$ \\ Dipartimento di Fisica Teorica, Università di Torino and INFN, Sezione di Torino, Via P. Giuria 1, 10125 Torino, Italy \\ P. Salati ${ }^{\S}$ \\ Laboratoire de Physique Théorique ENSLAPP, Boîte Postal 110, F-74941 Annecy-le-Vieux Cedex, France \\ (Received 16 April 1998; revised manuscript received 17 June 1998; published 12 November 1998)
}

\begin{abstract}
We analyze the data of the low-energy cosmic-ray $\bar{p}$ spectrum, recently published by the BESS Collaboration, in terms of newly calculated fluxes for secondary antiprotons and for a possible contribution of an exotic signal due to neutralino annihilation in the galactic halo. We single out the relevant supersymmetric configurations and discuss their explorability with experiments of direct search for dark matter particles and at accelerators. We discuss how future measurements with the alpha magnetic spectrometer on the shuttle flight may disentangle the possible neutralino-induced contribution from the secondary one. [S0556-2821(98)02020-7]

PACS number(s): 95.35.+d, 14.20.Dh, 14.80.Ly, 98.70.Sa
\end{abstract}

\section{INTRODUCTION}

A recent analysis [1] of the data collected by the balloonborne BESS spectrometer on cosmic-ray antiprotons during its flight in 1995 (hereafter referred to as BESS95 data) has provided the most detailed information on the low-energy cosmic-ray $\bar{p}$ 's spectrum currently available: 43 antiprotons have been detected, grouped in 5 narrow energy windows over the total kinetic-energy range $180 \mathrm{MeV} \leqslant T_{\bar{p}}$ $\leqslant 1.4 \mathrm{GeV}$. With this experiment the total number of measured cosmic-ray antiprotons in balloon-borne detectors over a period of more than 20 years [2-6] has more than doubled. Most remarkably, the BESS95 data provide very useful information over the low-energy part of the $\bar{p}$ flux, where a possible distortion of the spectrum expected for secondary $\bar{p}$ 's (i.e., antiprotons created by interactions of primary cosmic-ray nuclei with the interstellar medium) may reveal the existence of cosmic-ray antiprotons of exotic origin (for instance, due to pair annihilation of relic particles in the galactic halo [7-9], to evaporation of primordial black holes $[9,10]$ or to cosmic strings [11]). In fact, a possible discrimination between primary (exotic) and secondary $\bar{p}$ 's is based on the different features of their low-energy spectra: in this energy regime $\left(T_{p} \lesssim 1 \mathrm{GeV}\right)$ interstellar (IS) secondary $\bar{p}$ spectrum is expected to drop off very markedly because of kinematical reasons [12], while exotic antiprotons show a milder fall off. However, as will be discussed later on, this discrimination power is somewhat hindered by solar modulation and by some other effects affecting particle diffusion in the Galaxy.

In Fig. 1 we report the cosmic-ray $\bar{p}$ flux at the top of the

\footnotetext{
*Email address: bottino@to.infn.it

†Email address: donato@to.infn.it

*Email address: fornengo@to.infn.it

${ }^{\S}$ Email address: salati@lapp.in2p3.fr
}

atmosphere (hereafter referred to as TOA flux) measured by BESS95 [1]. For experimental data referring to other measurements with much less statistics see Refs. [2-6]. Also displayed in Fig. 1 are the minimal, median and maximal fluxes expected for secondary antiprotons at the time of the BESS95 data taking. These fluxes have been derived with a procedure which is described in detail in Secs. II-V.

A comparison of the BESS95 data with the theoretically expected fluxes for secondary $\bar{p}$ 's, as displayed in Fig. 1, leads to the following considerations: (i) the experimental data are consistent with the theoretically expected secondary

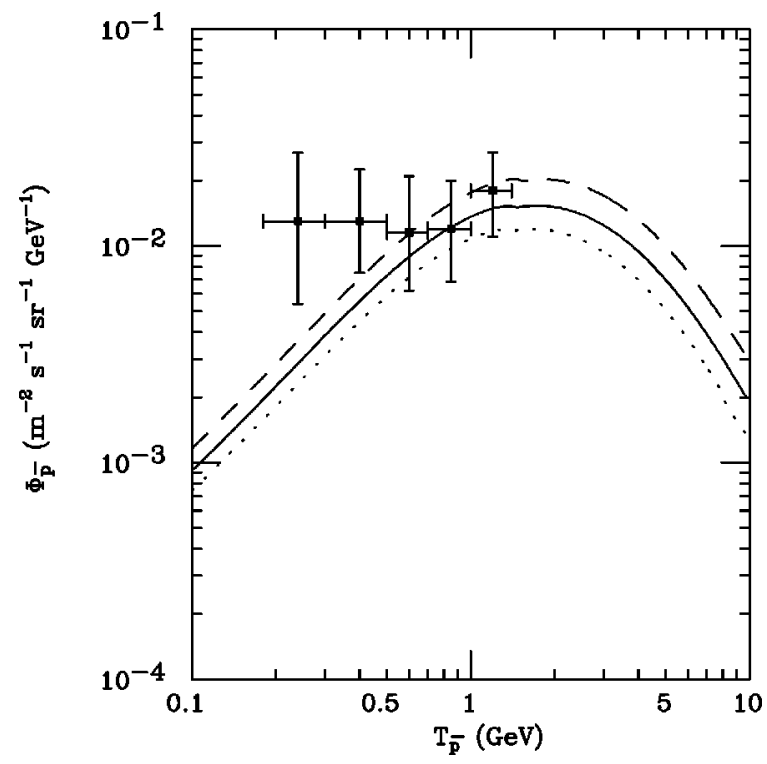

FIG. 1. TOA antiproton flux as a function of the antiproton kinetic energy. The experimental points are the BESS95 data [1]. The curves are the median (solid line), minimal (dotted line), and maximal (dashed line) secondary TOA fluxes obtained from the median, minimal, and maximal IS primary proton fluxes, as discussed in Sec. II. The TOA fluxes have been solar modulated for the time of the BESS95 measurement. 
flux, within the experimental errors and the theoretical uncertainties, however, (ii) the experimental flux seems to be suggestive of a flatter behavior, as compared to the one expected for secondaries $\bar{p}$ 's. Thus, natural questions arise, such as (a) how much room for exotic $\bar{p}$ 's would there be in the BESS95 data, for instance in case the secondary flux is approximately given by the median estimate of Fig. 1, (b) how consistent with the current theoretical models would be the interpretation of the BESS95 data in terms of a fractional presence of exotic antiprotons, and (c) how might this interpretation be checked by means of independent experiments? In the present paper we address these questions within an interpretation of a possible excess of $\bar{p}$ 's at low energies in terms of primary antiprotons generated by relic neutralinos in the galactic halo [13].

The present analysis [14] is mostly meant as a clarification of many theoretical points which will be even more crucial, when much more statistically significant experimental information on low-energy cosmic-ray antiprotons will be made available by forthcoming experiments: AMS on the precursor Shuttle flight in May 1998 and on the International Space Station Alpha (ISSA) in January 2002 [15], the satellite-borne PAMELA experiment [16], and balloon-borne measurements [17].

Our paper is organized as follows. In Sec. II we discuss the cosmic-ray IS proton spectrum which will be subsequently employed in deriving the secondary antiprotons. In the same section we also illustrate how we treat the solar modulation to connect the IS spectra to the corresponding TOA fluxes. In Sec. III we discuss the sources of cosmic antiprotons, both of primary and of secondary origins. Cosmic-ray diffusion properties are derived in Sec. IV; the TOA $\bar{p}$ spectra are given in Sec. V. In Sec. VI we compare our theoretical fluxes with the BESS95 data and single out the neutralino configurations which may be relevant for the present problem. Sections VII and VIII are devoted to an analysis on how these supersymmetric configurations can be explored by direct searches for relic neutralinos and by experimental investigation at accelerators. Conclusions and perspectives in terms of the forthcoming measurements of low-energy cosmic-ray $\bar{p}$ 's are illustrated in Sec. IX.

\section{COSMIC-RAY PROTON SPECTRUM}

We first have to fix the primary IS cosmic-ray proton spectrum, since we need it for the evaluation of the secondary $\bar{p}$ 's. The IS cosmic-ray proton spectrum is derived by assuming for it appropriate parametrizations and by fitting their corresponding solar-modulated expressions to the TOA experimental fluxes.

Measurements of the TOA spectra have always suffered from large uncertainties, as discussed for instance in Ref. [18]. In the present paper we use the two most recent highstatistics measurements of the TOA proton spectrum: the one reported by the IMAX Collaboration on the basis of a balloon flight in 1992 [19], the other given by the CAPRICE Collaboration based on data collected during a balloon flight in 1994 [20]. These two fluxes are reported in Fig. 2.

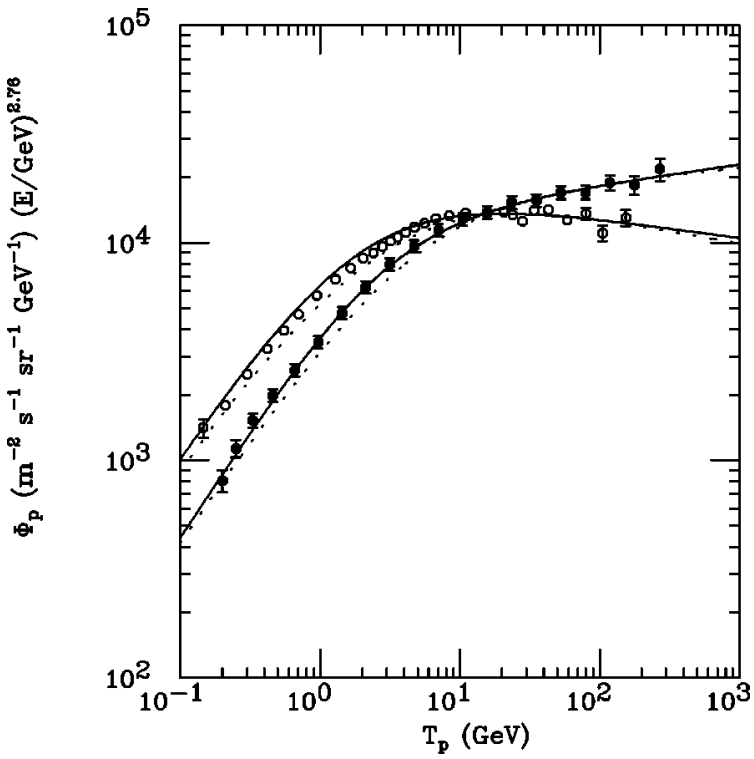

FIG. 2. TOA spectra of IMAX (full circles) [19] and of CAPRICE (open circles) [20] with our best-fit curves with parametrization of Eq. (1) (solid lines) and Eq. (2) (dotted lines). (The error bars on the data are not shown when they are smaller than the dimension of the circles.)

For the IS proton spectrum we have used two different parametrizations: one in terms of the total proton energy $E_{p}=T_{p}+m_{p}$

$$
\Phi_{p}^{\mathrm{IS}}\left(T_{p}\right)=A \beta\left(E_{p} / \mathrm{GeV}\right)^{-\alpha} \frac{\text { protons }}{\mathrm{m}^{2} \mathrm{~s} \text { sr } \mathrm{GeV}},
$$

the other in terms of momentum $p$ (equivalent to rigidity for protons),

$$
\Phi_{p}^{\mathrm{IS}}\left(T_{p}\right)=B \beta^{-1}(p / \mathrm{GeV})^{-\gamma} \frac{\text { protons }}{\mathrm{m}^{2} \mathrm{~s} \text { sr } \mathrm{GeV}},
$$

where $\beta=p / E_{p}$. For the solar modulation effect we have employed the Perko method [21], where the solar-modulated flux is given by

$$
\Phi^{\mathrm{TOA}}(T)=\frac{T^{2}+2 m_{p} T}{T_{\mathrm{IS}}^{2}+2 m_{p} T_{\mathrm{IS}}} \Phi^{\mathrm{IS}}\left(T_{\mathrm{IS}}\right) .
$$

The kinetic energies $T$ and $T_{\text {IS }}$ are simply related by $T_{\text {IS }}$ $=T+\Delta$, when $T \geqslant T_{\text {cut }}$ and by a more complicated relation otherwise [21]. Thus, this solar-modulation recipe is fully defined, once the values for the two parameters $\Delta$ and $T_{\text {cut }}$ are given.

The results of our best fits to the data of Refs. $[19,20]$ are reported in Table I in terms of the parameters of expressions (1) and (2) and of the solar-modulation parameter $\Delta$. The first and third sets of values for parameters $A, \alpha, \Delta$ [for expression (1)] and $B, \gamma, \Delta$ [for expression (2)] refer to three- 
TABLE I. Values of the parameters in the expressions (1) and (2) for the IS proton flux and of the solar-modulation parameter $\Delta$. These values are obtained by best-fitting the data of Refs. [19,20] with Eqs. (1) and (2), either over the entire energy range or only over the high-energy $\left(T_{p}>20 \mathrm{GeV}\right)$ range. First and third sets of values refer to three-parameter fits [with Eqs. (1) and (2), respectively], second and fourth sets refer to two-parameter fits at fixed $\Delta$ [with Eqs. (1) and (2), respectively].

\begin{tabular}{lccc}
\hline \hline & IMAX & CAPRICE & Comments \\
\hline$A$ & $12300 \pm 1700$ & $17600 \pm 500$ & entire energy \\
$\alpha$ & $2.67 \pm 0.03$ & $2.81 \pm 0.01$ & range \\
$\Delta$ & $510 \pm 40$ & $390 \pm 5$ & \\
$A$ & $12300 \pm 3000$ & $19600 \pm 3000$ & \\
$\alpha$ & $2.67 \pm 0.06$ & $2.85 \pm 0.04$ & $T_{p}>20 \mathrm{GeV}$ \\
$\Delta$ & 510 (fixed) & 390 (fixed) & \\
$B$ & $16200 \pm 2000$ & $26000 \pm 1200$ & entire energy \\
$\gamma$ & $2.73 \pm 0.03$ & $2.91 \pm 0.02$ & range \\
$\Delta$ & $795 \pm 35$ & $710 \pm 10$ & \\
$B$ & $13700 \pm 4100$ & $22800 \pm 3700$ & \\
$\gamma$ & $2.69 \pm 0.06$ & $2.88 \pm 0.04$ & $T_{p}>20 \mathrm{GeV}$ \\
$\Delta$ & 795 (fixed) & 710 (fixed) & \\
\hline \hline
\end{tabular}

parameter fits over the entire energy range of the experimental data. These fits are mainly meant to fix the solarmodulation parameter $\Delta$, since the low-energy part of the spectra is strongly dependent on the effect of solar modulation. The second and fourth sets of values refer to twoparameter fits (at fixed $\Delta)$ in the high energy range $\left(T_{p}\right.$ $\geqslant 20 \mathrm{GeV}$ ), where the solar modulation effect is less sizeable, however, not negligible, and therefore the proper parameters of the IS flux (normalization and spectral index) can be determined more confidently. The best-fit values for $T_{\text {cut }}$ turn out to be always smaller than the value corresponding to the lowest $T$ considered in the fit (i.e., $T_{\text {cut }}<0.1 \mathrm{GeV}$ ). This is consistent with the determination of the cutoff rigidity of the diffusion coefficient in the heliosphere $[22,23]$.

In Fig. 2 we display the curves of the best fits to the data of Refs. $[19,20]$ with the parametrizations of Eq. (1) (solid lines) and Eq. (2) (dotted lines). Both parametrizations for the IS flux provide good fits to the data of both experiments. The goodness of these fits at low energies indicates that the evaluation of solar modulation as described in Eq. (3) is appropiate.

From the values reported in Table I we notice that even using various parametric forms for the IS proton spectrum, the data of the two experiments of Refs. $[19,20]$ do not lead to a set of central values for the parameters mutually compatible within their uncertainties. This difference can be considered as due to systematics in the measurement of the proton spectra. The presence of systematic effects is apparent from the different behavior of the high-energy part of the experimental spectra, where the solar modulation does not sizeably affect the proton flux. In order to deal with the difference in the fits, the calculation of the secondary $\bar{p}$ 's is performed by defining a median cosmic-ray proton flux and an uncertainty band which takes into account the uncertainties coming from both sets of the experimental data. The

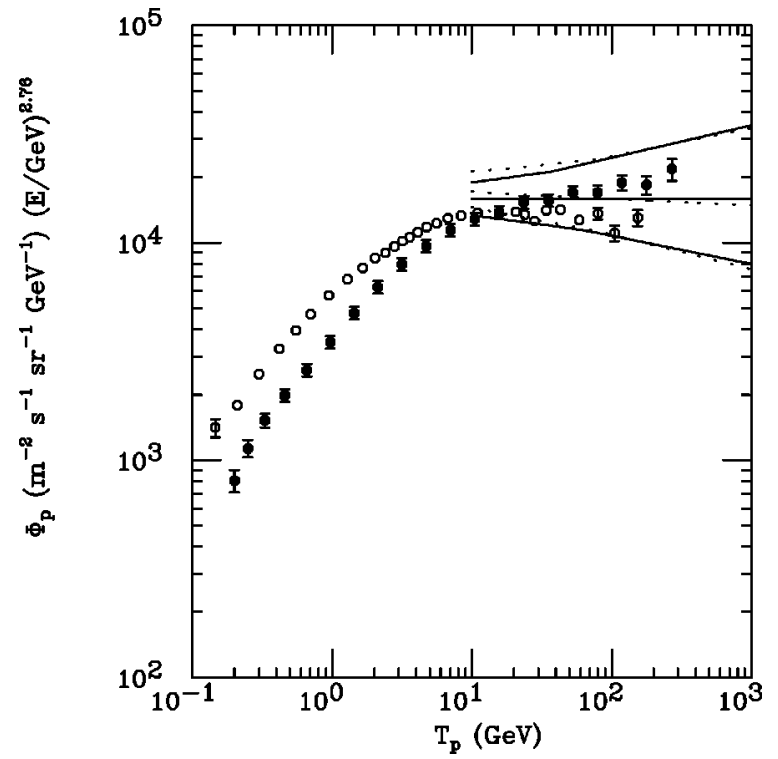

FIG. 3. TOA spectra of IMAX (full circles) [19] and of CAPRICE (open circles) [20]. The solid lines denote the median, minimal, and maximal IS proton fluxes obtained with parametrization of Eq. (1). The dotted lines denote the median, minimal, and maximal IS proton fluxes obtained with parametrization of Eq. (2). The minimal and maximal fluxes delimit our estimated uncertainty bands, as discussed in Sec. II. (The error bars on the data are not shown when they are smaller than the dimension of the circles.)

median proton flux is obtained by assigning to the parameters $A$ and $\alpha$ (and $B$ and $\gamma$, respectively) the averages of their central values obtained in the fits to the data of the two experiments for $T_{p} \geqslant 20 \mathrm{GeV}$. For instance, in the case of the parametrization of Eq. (1), the median proton flux is

$$
\Phi_{p}^{\mathrm{IS}}\left(T_{p}\right)=15,950 \beta\left(E_{p} / \mathrm{GeV}\right)^{-2.76} \frac{\text { protons }}{\mathrm{m}^{2} \mathrm{~s} \text { sr } \mathrm{GeV}} .
$$

A corresponding expression is obtained for Eq. (2) with $B=18,250$ and $\gamma=2.79$. For each parametrization [Eq. (1) and Eq. (2)] the uncertainty band around the median value is determined by the overlap of the 1- $\sigma$ error bands of the fits to the two sets of experimental data. The ensuing uncertainty band determines an upper and a lower proton flux which we use to determine the uncertainty in the secondary $\bar{p}$ flux arising from uncertainty in the primary proton flux.

The minimal, median and maximal IS proton fluxes are displayed in Fig. 3 together with the experimental TOA spectra of Refs. $[19,20]$. The solid lines refer to the parametrization of Eq. (1), the dotted lines stand for Eq. (2). We notice that both parametrizations are practically equivalent for the evaluation of the secondary antiproton flux. We choose to perform all subsequent calculations by employing the parametrization of Eq. (1), which is slightly preferable on the basis of the following features: (i) the fractional difference in the fit parameters between IMAX and CAPRICE are smaller for parametrization of Eq. (1); (ii) the fit parameters obtained with Eq. (1) vary less than for Eq. (2) when the fit over the whole energy range is compared to the high-energy 
fit. An additional good feature of Eq. (1) is related to our fits to the TOA proton fluxes of Ref. [24] which we will perform in Sec. $\mathrm{V}$ in order to determine the values of the solar modulation parameter $\Delta$ at minima and maxima of the solar cycle. In this case, the use of Eq. (1) provides a much better statistical agreement than Eq. (2).

As a final comment, we notice from Table I that the parametrization of Eq. (2) systematically provides larger values for the solar-modulation parameter $\Delta$ as compared to the ones obtained using the parametrization of Eq. (1). This is due to the steeper behavior at low energies of the function of Eq. (2) with respect to Eq. (1). The same approach used here in order to modulate the proton flux will be adopted in Sec. $\mathrm{V}$ to calculate the effect of solar modulation on the primary and secondary antiproton spectra. Different values of $\Delta$ have the consequence to generate different TOA antiproton fluxes. This will be explicitly discussed in Sec. V.

\section{PRODUCTION OF ANTIPROTONS IN THE GALAXY}

\section{A. Secondaries $\bar{p}$ 's}

Cosmic-ray protons interact with the interstellar material that mostly spreads in the galactic disk. This conventional spallation is actually a background to a hypothetical supersymmetric antiproton signal. It needs therefore to be carefully estimated, especially at low energies where new measurements are expected. The corresponding source term is given by the convolution between the antiproton production cross section and the interstellar proton energy spectrum as

$$
q_{\bar{p}}^{\mathrm{disk}}(r)=\int_{E_{p}^{0}}^{+\infty} \frac{d \sigma_{p \mathrm{H} \rightarrow \bar{p}}}{d E_{\bar{p}}^{-}}\left\{E_{p} \rightarrow E_{p}^{-}\right\} n_{\mathrm{H}} v_{p} \psi_{p}\left(r, E_{p}\right) d E_{p},
$$

where $n_{\mathrm{H}}$ is the hydrogen density in the disk, $v_{p}$ the proton velocity, and $\psi_{p}$ is the proton density per energy bin at distance $r$ from the galactic center in the galactic frame. The collision takes place between an incoming high-energy proton with a hydrogen atom at rest, lying in the gaseous HI and HII clouds of the galactic ridge. The proton energy is denoted by $E_{p}$. It is larger than the threshold $E_{p}^{0}=7 \mathrm{~m}$. That spallation reaction may generate an antiproton with energy $E_{\bar{p}}$. The relevant differential production cross section is the sum over the angle $\theta$ between the incoming proton and the produced antiproton momentum

$$
\begin{aligned}
\frac{d \sigma_{p \mathrm{H} \rightarrow \bar{p}}}{d E_{\bar{p}}}\left\{E_{p} \rightarrow E_{\bar{p}}^{-}\right\}= & \left.2 \pi P_{\bar{p}} \int_{0}^{\theta_{\max }} E_{\bar{p}}^{-} \frac{d^{3} \sigma}{d^{3} P_{\bar{p}}^{-}}\right|_{\mathrm{LI}} \\
& \times d(-\cos \theta),
\end{aligned}
$$

where $P_{\bar{p}}=\sqrt{E_{\bar{p}}^{2}-m^{2}}$. That integral is carried out in the galactic frame, at fixed antiproton energy $E_{p}^{-}$. The proton energy determines the center of mass frame (CMF) energy $\sqrt{s}=\left\{2 m\left(E_{p}+m\right)\right\}^{1 / 2}$. The latter sets in turn the maximal CMF energy $E_{p}^{*}$ max which the antiproton may carry away once it is produced

$$
E_{\bar{p} \max }^{*}=\frac{s-8 m^{2}}{2 \sqrt{s}}
$$

The range of angles $\theta$ over which the integral in Eq. (6) is performed is set by the requirement that the CMF antiproton energy $E_{p}^{*}$ should not exceed the maximal value $E_{p \text { max }}^{*}$ implied by kinematics. The Lorentz invariant antiproton production cross section $E_{\bar{p}} d^{3} \sigma / d^{3} P_{\bar{p}}$ has been parametrized by Tan and $\mathrm{Ng}$ [25] as a function of the transverse and longitudinal antiproton CMF momenta $P_{\bar{p} T}^{*}$ and $P_{\bar{p} L}^{*}$. We refer the interested reader to this analysis. The transverse momentum in the CMF is equal to $P_{\bar{p}}^{*}=P_{p}^{-} \sin \theta$ while the longitudinal momentum $P_{\bar{p} L}^{*}$ obtains from the component $P_{\bar{p}} \cos \theta$ after a Lorentz boost from the galactic frame to the CMF of the reaction. Note finally that the antiproton production integral in Eq. (5) should be a priori performed everywhere in the confining magnetic fields of the galactic disk. It actually involves the interstellar proton flux $\Phi_{p}$ which depends on the location $r$.

\section{B. $\bar{p}$ 's from neutralino annihilation}

The differential rate per unit volume and unit time for the production of $\bar{p}$ 's from $\chi-\chi$ annihilation is defined as

$$
q_{\bar{p}}^{\operatorname{SUSY}}\left(T_{\bar{p}}^{-}\right) \equiv \frac{d S\left(T_{\bar{p}}^{-}\right)}{d T_{\bar{p}}}=\left\langle\sigma_{\mathrm{ann}} v\right\rangle g\left(T_{\bar{p}}^{-}\right)\left(\frac{\rho_{\chi}(r, z)}{m_{\chi}}\right)^{2},
$$

where $\left\langle\sigma_{\mathrm{ann}} v\right\rangle$ denotes the average over the galactic velocity distribution function of neutralino pair annihilation cross section $\sigma_{\text {ann }}$ multiplied by the relative velocity $v$ of the annihilating particles, $m_{\chi}$ is the neutralino mass; $g\left(T_{\bar{p}}\right)$ denotes the $\bar{p}$ differential spectrum

$$
g\left(T_{\bar{p}}^{-}\right) \equiv \frac{1}{\sigma_{\mathrm{ann}}} \frac{d \sigma_{\mathrm{ann}}(\chi \chi \rightarrow \bar{p}+X)}{d T_{\bar{p}}^{-}}=\sum_{F, h} B_{\chi h}^{(F)} \frac{d N_{\bar{p}}^{h}}{d T_{\bar{p}}^{-}},
$$

where $F$ indicates the $\chi-\chi$ annihilation final states, $B_{\chi h}^{(F)}$ is the branching ratio into quarks or gluons $h$ in the channel $F$, and $d N_{\bar{p}}^{h} / d T_{\bar{p}}^{-}$is the differential energy distribution of the antiprotons generated by hadronization of quarks and gluons. In Eq. (8), $\rho_{\chi}(r, z)$ is the mass distribution function of neutralinos in the galactic halo. Here we consider the possibility that the halo is spheroidal and we parametrize $\rho_{\chi}(r, z)$ as a function of the radial distance $r$ from the galactic center in the galactic plane and of the vertical distance $z$ from the galactic plane

$$
\rho_{\chi}(r, z)=\rho_{\chi}^{0} \frac{a^{2}+r_{\odot}^{2}}{a^{2}+r^{2}+z^{2} / f^{2}},
$$

where $a$ is the core radius of the halo, $r_{\odot}$ is the distance of the Sun from the galactic center, and $f$ is a parameter which describes the flattening of the halo. Here we take the values $a=3.5 \mathrm{kpc}, r_{\odot}=8 \mathrm{kpc}$. For $f$, which in principle may be in the range $0.1 \leqslant f \leqslant 1$, we use the two representative values 
$f=0.5,1$ [26]. The quantity $\rho_{\chi}^{0}$ denotes the local value of the neutralino matter density. We factorize it as $\rho_{\chi}^{0}=\xi \rho_{l}$, where $\rho_{l}$ is the total local dark matter density. Here $\xi$ is evaluated as $\xi=\min \left[1, \Omega_{\chi} h^{2} /\left(\Omega h^{2}\right)_{\min }\right]$, where $\left(\Omega h^{2}\right)_{\min }$ is a minimal value compatible with observational data and with largescale structure calculations [27]. All the results of this paper refer to the choice $\left(\Omega h^{2}\right)_{\min }=0.03$. The neutralino relic density $\Omega_{\chi} h^{2}$ is calculated as a function of the supersymmetric parameters as described in Ref. [28]. As for the value $\rho_{l}$ of the total dark matter density, this is calculated by taking into account the contribution given by the matter density of Eq. (10) to the local rotational velocity. For instance, in the case of a spherical halo $(f=1)$, a value of $\rho_{l}=0.4 \mathrm{GeV} \mathrm{cm}^{-3}$ is obtained. When $f<1$ (oblate spheroidal distribution), $\rho_{l}$ is given by $[29,30]$

$$
\rho_{l}(f)=\rho_{l}(f=1) \frac{\sqrt{1-f^{2}}}{f \arcsin \sqrt{1-f^{2}}} .
$$

All the quantities depending on the supersymmetric parameters have been calculated in the framework of the minimal supersymmetric extension of the standard model (MSSM) [31], where the neutralino is defined as the lowestmass linear superposition of photino $(\tilde{\gamma})$, zino $(\widetilde{Z})$, and the two Higgsino states $\left(\tilde{H}_{1}^{\circ}, \tilde{H}_{2}^{\circ}\right)$

$$
\chi \equiv a_{1} \tilde{\gamma}+a_{2} \widetilde{Z}+a_{3} \tilde{H}_{1}^{\circ}+a_{4} \tilde{H}_{2}^{\circ} .
$$

For the evaluation of the averaged annihilation cross section $\left\langle\sigma_{\mathrm{ann}} v\right\rangle$ we have followed the procedure outlined in Ref. [8]. We have considered all the tree-level diagrams which are responsible of neutralino annihilation and which are relevant to $\bar{p}$ production, namely, annihilation into quark-antiquark pairs, into gauge bosons, into a Higgs boson pair, and into a Higgs and a gauge boson. For each final state we have considered all the relevant Feynman diagrams, which involve the exchange of Higgs and $Z$ bosons in the $s$ channel and the exchange of squarks, neutralinos, and charginos in the $t$ and $u$ channels. Finally, we have included the one-loop diagrams which produce a two-gluon final state. For this annihilation channel, we have used the recent results of Ref. [32].

The $\bar{p}$ differential distribution $g\left(T_{\bar{p}}\right)$ has been evaluated as discussed in Ref. [8]. Here we only recall that we have calculated the branching ratios $B_{\chi h}^{(F)}$ for all annihilation final states which may produce $\bar{p}$ 's, dividing these states into two categories: (i) direct production of quarks and gluons, (ii) generation of quarks through intermediate production of Higgs bosons, gauge bosons, and $t$ quarks. In order to obtain the distributions $d N_{\bar{p}}^{h} / d T_{\bar{p}}^{-}$the hadronization of quarks and gluons has been evaluated by using the Monte Carlo code JETSET 7.2 [33]. For the top quark, we have considered it to decay before hadronization.

We summarize now the main features of the MSSM scheme we employ here. The MSSM is defined at the electroweak scale as a straightforward supersymmetric extension of the standard model. The Higgs sector consists of two Higgs doublets $H_{1}$ and $H_{2}$, which define two free param- eters: the ratio of the two vacuum expectation values $\tan \beta$ $\equiv\left\langle H_{2}\right\rangle /\left\langle H_{1}\right\rangle$ and the mass of one of the three neutral physical Higgs fields; we choose as a free parameter the mass $m_{A}$ of the neutral pseudoscalar Higgs. The other parameters of the model are contained in the superpotential, which includes all the Yukawa couplings and the Higgs-mixing term $\mu H_{1} H_{2}$, and in the soft-breaking Lagrangian, which includes the trilinear and bilinear breaking parameters and the soft gaugino and scalar mass terms. In order to deal with a manageable model, we impose the following usual relations among the parameters at the electroweak scale: (i) all trilinear parameters are set to zero except those of the third family, which are unified to a common value $A$; (ii) all squarks and sleptons soft-mass parameters are taken as degenerate, $m_{\tilde{l}_{i}}=m_{\tilde{q}_{i}} \equiv m_{0}$; (iii) the gaugino masses are assumed to unify at $M_{\mathrm{GUT}}$, and this implies that the $\mathrm{U}(1)$ and $\mathrm{SU}(2)$ gaugino masses are related at the electroweak scale by $M_{1}$ $=(5 / 3) \tan ^{2} \theta_{W} M_{2}$.

When all these conditions are imposed, the supersymmetric parameter space is completely described by six independent parameters, which we choose to be $M_{2}, \mu, \tan \beta, m_{A}$, $m_{0}, A$. In our analyses, we vary them in the following ranges: $10 \mathrm{GeV} \leqslant M_{2} \leqslant 500 \mathrm{GeV}$ (21 steps over a linear grid); $10 \mathrm{GeV} \leqslant|\mu| \leqslant 500 \mathrm{GeV}$ (21 steps, linear grid); $75 \mathrm{GeV} \leqslant m_{A} \leqslant 500 \mathrm{GeV}$ (15 steps, logarithmic grid); $100 \mathrm{GeV} \leqslant m_{0} \leqslant 500 \mathrm{GeV}$ (5 steps, linear grid); $-3 \leqslant \mathrm{~A} \leqslant$ +3 (5 steps, linear grid); $1.01 \leqslant \tan \beta \leqslant 50$ (15 steps, logarithmic grid).

The supersymmetric parameter space is constrained by all the experimental limits obtained from accelerators on supersymmetric and Higgs searches. The latest CERN $e^{+} e^{-}$collider LEP2 data on Higgs, neutralino, chargino, and sfermion masses [34] and the constraints due to the $b \rightarrow s+\gamma$ process [35] are imposed. Moreover, the request for the neutralino to be the lightest supersymmetric particle (LSP) implies that regions where the gluino or squarks or sleptons are lighter than the neutralino are excluded. A further constraint is imposed by requiring that all the supersymmetric configurations which provide a neutralino relic abundance are in accordance with the cosmological bound $\Omega_{\chi} h^{2} \leqslant 0.7$.

\section{DIFFUSION OF COSMIC RAYS INSIDE THE GALAXY}

The propagation of cosmic rays inside the Galaxy has been considered in the framework of a two-zone diffusion model. We have followed here the same analysis as Webber, Lee, and Gupta [36]. The Milky Way is pictured as a thin disk, $200 \mathrm{pc}$ across, that extends radially up to $R=20 \mathrm{kpc}$ from the galactic center. That ridge lies between two extended layers $\sim 3 \mathrm{kpc}$ thick, where cosmic rays diffuse in erratic magnetic fields. Mere diffusion governs the propagation of the particles in the disk and in the confinement regions that extend on either side. Assuming that steady state holds, the proton density $\psi_{p}$, per energy bin, at some location $r$ and $z$, is given by

$$
\frac{\partial \psi_{p}}{\partial t}=0=\vec{\nabla} \cdot\left(K \vec{\nabla} \psi_{p}\right)+2 h \delta(z) q(r)-2 h \delta(z) \Gamma_{p} \psi_{p} .
$$


The diffusion coefficient $K$ is assumed to be essentially independent of the nature of the species that propagate throughout the Galaxy. It increases with rigidity $\mathcal{R}$ according to the relation

$$
K(\mathcal{R})=K_{0}\left(1+\frac{\mathcal{R}}{\mathcal{R}^{0}}\right)^{0.6},
$$

where $K_{0}=6 \times 10^{27} \mathrm{~cm}^{2} \mathrm{~s}^{-1}$ and $\mathcal{R}_{0}=1 \mathrm{GV}$. Below that critical value, the diffusion coefficient stays constant while above $1 \mathrm{GV}$, it increases as $\mathcal{R}^{0.6}$. Sources are located in the galactic ridge at $z=0$. Their radial profile is inferred from the survey by Lyne, Manchester, and Taylor of the galactic distribution of stellar remnants and pulsars [37] with $q(r, 0)$ $\propto \rho^{a} \exp (-b \rho)$ where $\rho=r / R, a=0.6$, and $b=3$. Finally, cosmic ray protons may interact with the interstellar gas. The latter is assumed to be concentrated in the disk. The probability per unit time that a proton collides with an interstellar hydrogen atom at rest is

$$
\Gamma_{p}=n_{\mathrm{H}} \sigma_{p \mathrm{H}}^{\mathrm{tot}} v_{p} .
$$

The hydrogen density $n_{\mathrm{H}}$ is assumed to be constant all over the disk. The value of $n_{\mathrm{H}}=1 \mathrm{~cm}^{-3}$ is basically consistent with measurements of the hydrogen column density derived from $\mathrm{HI}$ and $\mathrm{CO}$ surveys. It implies in particular a maximal value of $\sim 9 \times 10^{22} \mathrm{H} \mathrm{cm}^{-2}$ to be compared to an average of $5 \times 10^{22} \mathrm{H} \mathrm{cm}^{-2}$ on the observations of the galactic center. The densest spot is inferred from $\mathrm{CO}$ measurements to reach a level of $\sim 1.4 \times 10^{23} \mathrm{H} \mathrm{cm}^{-2}$. The total interaction cross section $\sigma_{p \mathrm{H}}^{\text {tot }}$ between the propagating high-energy protons and the hydrogen atoms of the interstellar medium has been borrowed from the work by Tan and $\mathrm{Ng}$ [38]. Above a kinetic energy of $3 \mathrm{GeV}$, it may be expressed as

$$
\sigma_{p \mathrm{H}}^{\mathrm{tot}}=(32.2 \mathrm{mb})\{1+0.0273 U\},
$$

where the parameter $U$ is defined as

$$
U=\ln \left(E_{p} / 200 \mathrm{GeV}\right) .
$$

Below $T_{p}=3 \mathrm{GeV}$, expression (16) needs to be divided by a low-energy correction factor equal to $1+0.00262 T_{p}{ }^{-C_{p}}$ where

$$
C_{p}=17.9+13.8 \ln T_{p}+4.41 \ln ^{2} T_{p} .
$$

The galactic disk is assumed to be infinitely thin, hence the factor $2 h \delta(z)$ in the diffusion equation (13), where $2 h$ $=200 \mathrm{pc}$ stands for the actual thickness of the ridge.

Because the Galaxy is axisymmetric, we can expand the proton density $\psi_{p}$ as a series of Bessel functions of zeroth order

$$
\psi_{p}(r, z)=\sum_{i=1}^{+\infty} \mathcal{P}_{i}(z) J_{0}\left(\alpha_{i} \rho\right),
$$

where $\rho=r / R$, while $\alpha_{i}$ is the $i$ th zero of the Bessel function $J_{0}$. The proton density is ensured to vanish at the radial boundary $r=R$ of the system. The Bessel transforms $\mathcal{P}_{i}$ must also drop to zero at the boundaries of the confinement regions, at a distance $L=3 \mathrm{kpc}$ from either side of the galactic disk. The distribution of cosmic ray sources may also be expanded as a series of Bessel functions

$$
q\left(r, E_{p}\right)=\sum_{i=1}^{+\infty} \frac{q_{i}}{2 h} Q_{\mathrm{tot}}\left(E_{p}\right) J_{0}\left(\alpha_{i} \rho\right),
$$

where $Q_{\mathrm{tot}}\left(E_{p}\right)$ stands for the total galactic rate of production, per energy bin, of cosmic ray protons with energy $E_{p}$. The Bessel transforms $q_{i}$ are readily inferred from the radial distribution of the sources in the galactic disk

$$
q_{i}=\frac{1}{\pi R^{2}} \frac{1}{J_{1}^{2}\left(\alpha_{i}\right)}\left\{\int_{0}^{1} q(\rho) J_{0}\left(\alpha_{i} \rho\right) d \rho^{2}\right\}\left\{\int_{0}^{1} q(\rho) d \rho^{2}\right\}^{-1} .
$$

Bessel expanding the diffusion equation (13) leads to simple differential relations which the functions $\mathcal{P}_{i}(z)$ satisfy. The latter are even functions of the height $z$ that vanish at the boundaries of the diffusion layers. Straightforward algebra leads to

$$
\mathcal{P}_{i}\left(z, E_{p}\right)=\frac{q_{i}}{A_{i}} Q_{\mathrm{tot}}\left(E_{p}\right) \sinh \left\{\frac{S_{i}}{2}(L-|z|)\right\} / \sinh \left\{\frac{S_{i} L}{2}\right\},
$$

where $S_{i}=2 \alpha_{i} / R$ and where the coefficients $A_{i}$ are defined by

$$
A_{i}=2 h \Gamma_{p}+K S_{i} \operatorname{coth}\left(\frac{S_{i} L}{2}\right) .
$$

Because the diffusion term dominates the behavior of the coefficients $A_{i}$, the proton energy spectrum does not vary much all over the Galaxy, except for a global normalization factor. In other words, the ratio of the proton fluxes taken at two different energies is quite insensitive to the location $M$, hence

$$
\Phi_{p}\left(M, E_{1}\right) / \Phi_{p}\left(M, E_{2}\right) \simeq \mathcal{P}_{i}\left(0, E_{1}\right) / \mathcal{P}_{i}\left(0, E_{2}\right) .
$$

This will turn out to be important when we compute the energy spectrum of secondary antiprotons.

The two-zone model is a refinement with respect to the old leaky box scheme. The confinement layers are necessary in order to account for the low abundance of the ${ }^{10} \mathrm{Be}$ unstable element with respect to its stable partner ${ }^{9} \mathrm{Be}$. The former nucleus has a half-lifetime of 1.6 million years (My) and plays the role of a chronometer. Observations indicate that cosmic rays are trapped in the magnetic fields of our Galaxy for approximately 100 My before they escape in the intergalactic medium. On the other hand, the amount of secondary light nuclei such as lithium, beryllium, and boron ( $\mathrm{Li}-\mathrm{Be}-\mathrm{B})$ is well explained by the spallation of primary carbon, oxygen, and nitrogen $(\mathrm{CNO})$ nuclei. The latter spend a mere $5 \mathrm{My}$ in the galactic plane where they cross a column density of $\sim 10 \mathrm{~g} \mathrm{~cm}^{-2}$. Cosmic rays are therefore confined most of the time in extended reservoirs above and beneath the matter ridge, where they just diffuse without inter- 
acting much with the scarce interstellar medium. We have estimated the grammage which the $\mathrm{CNO}$ elements cross during their journey inside the galactic disk. Their distribution is inferred in just the same way as for the protons. The average electric charge per nucleon is now $1 / 2$ instead of 1 for the protons, hence a slightly modified relationship between the kinetic energy per nucleon and the rigidity of the nucleus under consideration. The grammage is defined as the product

$$
\Lambda_{e}=n_{\mathrm{H}} v_{\mathrm{N}} \tau_{\text {disk }},
$$

where the confinement time in the disk alone is denoted by $\tau_{\text {disk }}$. The escape length $\Lambda_{e}$ is expressed in units of $\mathrm{g} \mathrm{cm}^{-2}$. Because cosmic rays either escape from the disk or interact with its gas, the total number $\mathcal{N}_{\mathrm{N}}^{\text {disk }}$ of particles contained in the galactic ridge satisfies the balance relation

$$
Q_{\mathrm{N}}=\frac{\mathcal{N}_{\mathrm{N}}^{\text {disk }}}{\tau_{\text {disk }}}+\Gamma_{\mathrm{N}} \mathcal{N}_{\mathrm{N}}^{\text {disk }}
$$

The rate $Q_{\mathrm{N}}$ at which the CNO primaries are produced is set equal to the sum of the escape rate from the galactic ridge and of the interaction rate with the interstellar gas. Notice that in the case of the two-zone model, the amount $\mathcal{N}_{\mathrm{N}}^{\text {disk }}$ of cosmic rays traveling in the disk alone may be expressed as the series

$$
\frac{\mathcal{N}_{\mathrm{N}}^{\text {disk }}}{Q_{\mathrm{N}}}=4 \pi h R^{2} \sum_{i=1}^{+\infty} \frac{q_{i}}{A_{i}} \frac{J_{1}\left(\alpha_{i}\right)}{\alpha_{i}} .
$$

In the coefficients $A_{i}$, the relevant cross section that accounts for the interactions of the $\mathrm{CNO}$ species with the interstellar hydrogen has been averaged at a mere $250 \mathrm{mb}$. In Fig. 4, an estimate of the grammage $\Lambda_{e}$ crossed by the CNO elements is presented as a function of the kinetic energy per nucleon (solid line). It reaches a maximum of $\sim 8 \mathrm{~g} \mathrm{~cm}^{-2}$ at $500 \mathrm{MeV} / n$. It decreases at low energies with the velocity. It also drops at high energies as a result of a better diffusion and hence a lower time of residence in the disk. The dashed curve refers to the grammage of the protons. At fixed kinetic energy, the diffusion coefficient is slightly smaller for these species than for heavier elements, hence a larger escape length $\Lambda_{e}$. Measurements of the ${ }^{2} \mathrm{H}$ abundance have been performed [39] from the Voyager probe at a distance of 23 $\mathrm{AU}$ and at energies lying between 20 and $50 \mathrm{MeV} / n$. With a solar modulation parameter of $\sim 360 \mathrm{MV}$, this translates into an energy of $\sim 230 \mathrm{MeV} / n$ in interstellar space. The analysis by Seo et al. of these data is well accounted for by the leaky box model using a grammage $\Lambda_{e}(\mathrm{~B} / \mathrm{C}) \sim 8 \mathrm{~g} \mathrm{~cm}^{-2}$. This is in excellent agreement with the results of our twozone model presented in Fig. 4, where the diffusion coefficient $K$ is given by relation (14). Ficenec et al. [40] have taken data on ${ }^{3} \mathrm{He}$ between $100 \mathrm{MeV} / n$ and $1.6 \mathrm{GeV} / n$. They conclude that the grammage of primary cosmic rays is well fitted by $\Lambda_{e}=(10.5+2.5-2.8) \beta \mathrm{g} \mathrm{cm}^{-2}$. The extreme values of that fit are featured by the dotted curves of Fig. 4 . Notice that the CNO grammage inferred from our two-zone model lies in the range of escape length delineated by the Ficenec et al. extreme values, for energies in interstellar

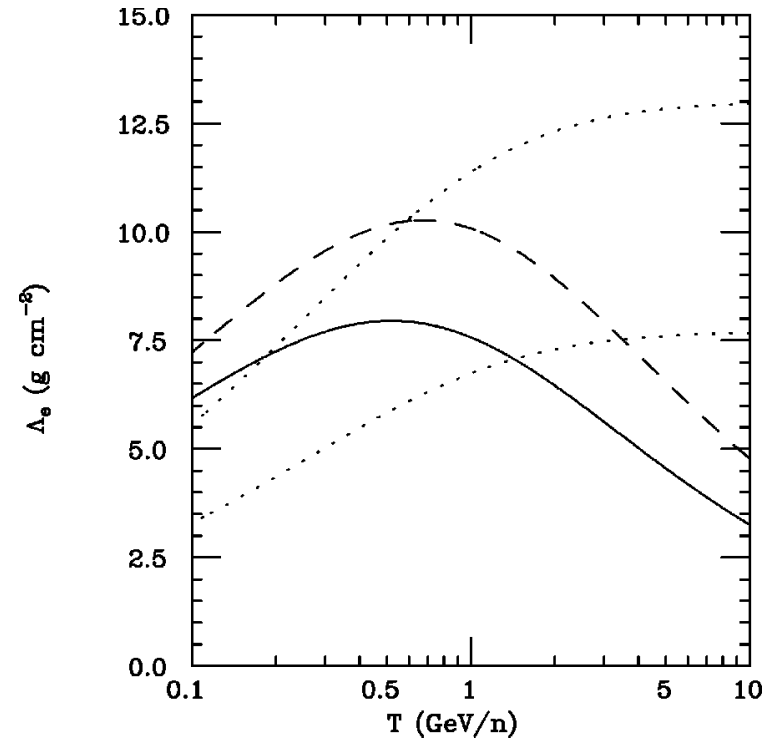

FIG. 4. The grammage $\Lambda_{e}$ of the CNO primary elements (solid) as inferred from a two-zone diffusion model of the propagation of cosmic rays in the Galaxy. It is plotted as a function of the kinetic energy per nucleon. The dashed curve features the grammage corresponding to protons while the dotted lines delineate the interval of escape lengths inferred from the Ficenec et al. [40] observations on ${ }^{3} \mathrm{He}$ at TOA energies comprised between $100 \mathrm{MeV} / n$ and $1.6 \mathrm{GeV} / n$.

space comprised between $200 \mathrm{MeV} / n$ and $1.5 \mathrm{GeV} / n$. The expression which we have adopted for the diffusion coefficient $K$ is therefore well supported by measurements [41] of the grammage encountered by primary $\mathrm{CNO}$ cosmic rays while they propagate within the galactic ridge. Comparison between our estimate of the CNO grammage with the Ficenec et al. range of values constrains the diffusion coefficient. We have required that the escape lengths should be compatible at least on half the energy interval of interest, i.e., between $200 \mathrm{MeV}$ and $1.4 \mathrm{GeV}$. This translates into the coefficient $K_{0}$ lying in the range $(4.5-7.8) \times 10^{27} \mathrm{~cm}^{2} \mathrm{~s}^{-1}$ while the critical rigidity $\mathcal{R}_{0}$ is comprised between 0.55 and $2 \mathrm{GV}$.

The propagation of antiprotons throughout the Galaxy follows the same trends as for the protons. We focus first on the species produced by the spallation of cosmic ray protons with the interstellar gas of the ridge. Their density $\psi_{p}^{-}$, per energy bin, follows the diffusion equation

$$
\begin{aligned}
\vec{\nabla} \cdot( & \left.K \vec{\nabla} \psi_{p}^{-}\right)-2 h \delta(z) \Gamma_{\bar{p}}^{-} \psi_{p}^{-} \\
& +2 h \delta(z) q_{\bar{p}}^{\text {disk }}(r)-2 h \delta(z) \frac{\partial}{\partial E}\left\{b(E) \psi_{p}^{-}\right\}=0,
\end{aligned}
$$

where steady state has once again been assumed. We recognize the usual diffusion term as well as the contribution due to the interactions of the antiproton cosmic rays with the matter of the disk. The total interaction cross section between antiprotons and the hydrogen atoms of the interstellar 
medium has also been borrowed from the analysis by Tan and $\mathrm{Ng}$ [38]. Above $T_{\bar{p}}^{-}=50 \mathrm{MeV}$, it may be parametrized as

$$
\sigma_{\bar{p} \mathrm{H}}^{\mathrm{tot}}=(24.7 \mathrm{mb}) \quad\left\{1+0.584 T_{\bar{p}}^{-0.115}+0.856 T_{\bar{p}}^{-0.566}\right\},
$$

where the antiproton kinetic energy $T_{\bar{p}}^{-}$is expressed in $\mathrm{GeV}$. The spallation source term has already been discussed in Sec. III A. It obtains from the convolution (5) of the antiproton production cross section with the proton energy spectrum. In order to simplify the calculations, we define the effective antiproton multiplicity

$$
N_{\bar{p}}^{\mathrm{eff}}\left(E_{\bar{p}}^{-}\right)=\frac{1}{\sigma_{p \mathrm{H}}^{\mathrm{tot}}\left(E_{\bar{p}}^{-}\right)} \int_{E_{p}^{0}}^{+\infty} \frac{d \sigma_{p \mathrm{H} \rightarrow \bar{p}}}{d E_{\bar{p}}^{-}}\left\{E_{p} \rightarrow E_{\bar{p}}^{-}\right\} \frac{\Phi_{p}\left(E_{p}\right)}{\Phi_{p}\left(E_{\bar{p}}^{-}\right)} d E_{p} .
$$

Because the ratio of the proton fluxes taken at two different energies does not depend on the location, the effective antiproton multiplicity $N_{\bar{p}}^{\text {eff }}$ is inferred to be only sensitive to the energy. It is therefore constant throughout the galactic ridge and may be computed once and for all as a function of the energy $E_{\bar{p}}$ of the produced antiproton before the diffusion equation (28) is solved. The spallation production term readily simplifies into

$$
q_{\bar{p}}^{\text {disk }}(r, E)=\sigma_{p \mathrm{H}}^{\mathrm{tot}}(E) N_{\bar{p}}^{\mathrm{eff}}(E) v_{p} n_{\mathrm{H}} \psi_{p}(r, E) .
$$

Under that form, it may be immediately expanded as the usual series of Bessel functions of zeroth order. The last term in relation (28) stands for the energy losses suffered by the antiproton cosmic rays while they propagate in the galactic disk. That term actually exists for any cosmic ray species. Because the particle fluxes do not significantly drop at low energies, this effect is in general neglected. Fluxes tend even to increase below $1 \mathrm{GeV}$. In the specific case of secondary antiprotons, that is no longer valid. Because a high-energy proton has very little chance to produce an antiproton at rest while colliding on a hydrogen atom, the secondary antiproton flux sharply drops when the energy decreases below $\sim 1 \mathrm{GeV}$. Energy losses tend to shift the antiproton spectrum towards lower energies with the effect of replenishing the low-energy tail with the more abundant species which had initially a higher energy. This process is understood here as a mere diffusion in energy space. The rate at which the antiproton energy varies $b\left(E_{\bar{p}}^{-}\right)=\dot{E}_{\bar{p}}$ takes into account two main effects. First, antiprotons may suffer from ionization losses while they travel across the interstellar gas. This mechanism yields the following contribution to the energy loss rate:

$b_{\text {ion }}(E)=-4 \pi r_{e}^{2} m_{e} c^{2} n_{\mathrm{H}} \frac{c}{\beta}\left\{\ln \left(\frac{2 m_{e} c^{2}}{E_{0}}\right)+\ln \left(\beta^{2} \gamma^{2}\right)-\beta^{2}\right\}$.

In molecular hydrogen, the ionization energy $E_{0}$ has been set equal to $19.2 \mathrm{eV}$; here $\gamma=E / m$. The classical radius of the electron is denoted by $r_{e}$ and the electron mass is $m_{e}$. Second, the dominant contribution to the energy losses arises from the elastic scatterings of high-energy antiprotons on the hydrogen atoms of the disk. This mechanism is a counterpart to the collision process whose rate is $\Gamma_{\bar{p}}$. An antiproton with initial energy $E_{1}$ ends up after such a collision in a final state with the lesser energy $E_{2}$. Elastic scatterings feed therefore the low-energy part of the antiproton distribution. They have been described here as if they induced a continuous change in the antiproton energy. Our assumption is correct on average, hence the contribution

$$
b_{\text {scat }}\left(E_{\bar{p}}^{-}\right)=-\frac{T_{\bar{p}}^{-}}{2}\left\{\sigma_{\bar{p} \mathrm{H}}^{\mathrm{el}}\left(E_{\bar{p}}^{-}\right) n_{\mathrm{H}} v_{p}^{-}\right\} .
$$

The elastic cross section $\sigma_{\bar{p}}^{\mathrm{el}}$ obtains from the difference $\sigma_{\bar{p} \mathrm{H}}^{\mathrm{tot}}-\sigma_{\bar{p} \mathrm{H}}^{\mathrm{an}}$ where the annihilation cross section is given by

$$
\sigma_{\bar{p} \mathrm{H}}^{\mathrm{an}}=(661 \mathrm{mb})\left\{1+0.0115 T_{\bar{p}}^{-0.774}-0.948 T_{\bar{p}}^{0.0151}\right\},
$$

between $100 \mathrm{MeV}$ and $12 \mathrm{GeV}$, i.e., the energy range under scrutiny here. Low-energy data are fairly consistent with an average energy loss approximately equal to a half of the initial antiproton kinetic energy [42].

The antiproton density $\psi_{p}^{-}$, per energy bin, may be Bessel transformed into the functions $\overline{\mathcal{P}}_{i}$ whose variations with height $z$ are given by

$$
\overline{\mathcal{P}}_{i}\left(E_{\bar{p}}^{-}, z\right)=\overline{\mathcal{P}}_{i}\left(E_{\bar{p}}^{-}, 0\right) \sinh \left\{\frac{S_{i}}{2}(L-|z|)\right\} / \sinh \left\{\frac{S_{i} L}{2}\right\} .
$$

In the galactic disk at $z=0$, the Bessel transforms $\overline{\mathcal{P}}_{i}\left(E_{\bar{p}}, 0\right)$ only depend on the antiproton energy $E_{\bar{p}}^{-}$. They actually satisfy a first order differential equation

$$
2 h \frac{\partial}{\partial E}\left(b \overline{\mathcal{P}}_{i}\right)+B_{i} \overline{\mathcal{P}}_{i}=\left.2 h\left(\sigma_{p \mathrm{H}}^{\mathrm{tot}} N_{\bar{p}}^{\mathrm{eff}} v_{p}\right)\right|_{E} n_{\mathrm{H}} \mathcal{P}_{i}(E, 0),
$$

which we have numerically solved for each order $i \leqslant 100$. At high energy, antiprotons are insensitive to the energy losses. Starting therefore from an unperturbed spectrum, we have decreased the kinetic energy from $10 \mathrm{GeV}$ down to $100 \mathrm{MeV}$ while integrating equation (36). The coefficients $B_{i}$ obtain from $A_{i}$ by replacing the rate $\Gamma_{p}$ by its antiproton counterpart $\Gamma_{\bar{p}}$. The abovementioned method has been applied to the case of the median proton flux (4) derived from the IMAX and CAPRICE measurements. The solid curve of Fig. 5 stands for the corresponding antiproton interstellar flux. Energy losses have been taken into account. This is not the case, however, for the dot-dashed line where the same proton spectrum has been assumed. Note that energy losses tend actually to replenish the low-energy part of the antiproton distribution. This effect is particularly evident at low energy. For $T_{\bar{p}} \sim 100 \mathrm{MeV}$, the antiproton flux increases by more 


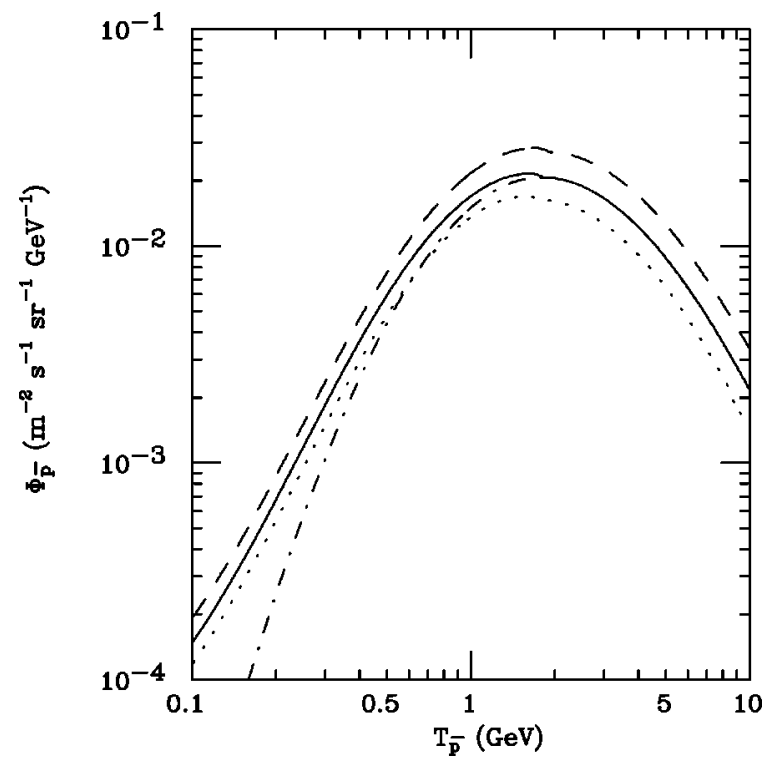

FIG. 5. IS secondary antiproton spectra as functions of the $\bar{p}$ kinetic energy. Solid, dotted, and dashed lines denote the fluxes obtained from the median, minimal, and maximal IS primary proton fluxes. The dot-dashed line denotes the median $\bar{p}$ spectrum, when the $\bar{p}$ energy losses are neglected.

than an order of magnitude when energy losses in the gaseous disk are considered. At larger energies, the upward shift of the spectrum is less sizeable. For an interstellar kinetic energy of $600 \mathrm{MeV}$, the increase has reduced to $\sim 30 \%$. Even in the case of minimal solar modulation, $600 \mathrm{MeV}$ in interstellar space translate into $T_{p}^{-} \sim 300 \mathrm{MeV}$. The dotted and dashed curves respectively stand for the antiproton spectra derived from the minimal and maximal IS proton flux discussed in Sec. II. The resulting relative uncertainty on the IS antiproton flux is $\sim \pm 25 \%$ for energies lying between 100 $\mathrm{MeV}$ and $1 \mathrm{GeV}$. It is fairly constant on that energy range. The range of allowed values for the diffusion coefficient introduces also an uncertainty into our estimate of the IS antiproton flux. When the critical rigidity $\mathcal{R}_{0}$ is varied from 0.55 to $2 \mathrm{GV}$, the latter changes by $\pm 16 \%$ at $1 \mathrm{GeV}$ and by $\pm 25 \%$ at $100 \mathrm{MeV}$. On the other hand, values of $K_{0}$ lying in the range $(4.5-7.8) \times 10^{27} \mathrm{~cm}^{2} \mathrm{~s}^{-1}$ translate into a relative uncertainty in the antiproton flux of order $\pm 45 \%$ at 100 $\mathrm{MeV}$, decreasing to $\pm 20 \%$ when the kinetic energy exceeds $600 \mathrm{MeV}$. In the previous analysis of Ref. [43], a critical rigidity $\mathcal{R}_{0}=3 \mathrm{GV}$ was assumed. That value is not compatible with the Ficenec et al. range of allowed CNO grammages. Note, however, that increasing $\mathcal{R}_{0}$ from 1 to $3 \mathrm{GV}$ results into a moderate increase of the IS antiproton flux by a mere $33 \%$ at $100 \mathrm{MeV}$ and $25 \%$ at $1 \mathrm{GeV}$. One should also keep in mind that energy loss was not included in that work.

We finally analyze the case of the antiprotons produced in the annihilations of neutralinos potentially concealed in the galactic halo. The production of these antiprotons from supersymmetric origin and their subsequent propagation from the remote regions of the dark matter halo back to the Earth has already been briefly mentioned in [43] and summarized in [44]. A more detailed discussion of that result follows.
The diffusion equation is quite similar to relation (28)

$$
\vec{\nabla} \cdot\left(K \vec{\nabla} \psi_{p}^{-}\right)-2 h \delta(z) \Gamma_{\bar{p}}^{-} \psi_{p}^{-}+q_{\bar{p}}^{-\mathrm{SUSY}}(r, z)=0 .
$$

Because the energy distribution of these supersymmetric antiprotons is fairly flat, energy losses in the disk should play a negligible role. They have not been considered here. The source term (8) has already been discussed in Sec. III B. The antiproton production extends now all over the Galaxy and not solely in the disk. The solution of the diffusion equation (37) follows, however, the same trends as for the previous cases. The antiproton energy distribution $\psi_{p}^{-}$may still be expanded as a series of its Bessel transforms $\overline{\mathcal{P}}_{i}\left(E_{\bar{p}}, z\right)$. Since energy losses are negligible, the latter obey the simple differential equation

$$
K\left\{\frac{d^{2} \overline{\mathcal{P}}_{i}}{d z^{2}}-\frac{\alpha_{i}^{2}}{R^{2}} \overline{\mathcal{P}}_{i}\right\}-2 h \delta(z) \Gamma_{p}-\overline{\mathcal{P}}_{i}+q_{i}^{\mathrm{SUSY}}(z)=0
$$

The Bessel transforms of the supersymmetric antiproton source distribution $q_{\bar{p}}^{\text {SUSY }}$ are defined as

$$
q_{i}^{\mathrm{SUSY}}(z)=\frac{1}{J_{1}^{2}\left(\alpha_{i}\right)} \int_{0}^{1} J_{0}\left(\alpha_{i} \rho\right) q_{\bar{p}}^{\mathrm{SUSY}}(r=\rho R, z) d \rho^{2} .
$$

Outside the galactic ridge, Eq. (38) simplifies even further into

$$
\frac{d^{2} \overline{\mathcal{P}}_{i}}{d z^{2}}-\frac{\alpha_{i}^{2}}{R^{2}} \overline{\mathcal{P}}_{i}+\frac{q_{i}^{\mathrm{SUSY}}(z)}{K}=0
$$

For positive values of the height $z$, the general solution may be expressed as

$$
\begin{aligned}
\overline{\mathcal{P}}_{i}(z>0)= & a_{i} \cosh \left(\frac{S_{i} z}{2}\right)+b_{i} \sinh \left(\frac{S_{i} z}{2}\right) \\
& +\frac{1}{K S_{i}} \int_{0}^{L} \exp \left(-S_{i}\left|z-z^{\prime}\right| / 2\right) q_{i}^{\mathrm{SUSY}}\left(z^{\prime}\right) d z^{\prime},
\end{aligned}
$$

where $S_{i}=2 \alpha_{i} / R$. Remember that the functions $\overline{\mathcal{P}}_{i}(z)$ are even since symmetry with respect to the galactic plane is assumed. We leave as an exercise the determination of the constants of integration $a_{i}$ and $b_{i}$. They obtain from the requirement that the Bessel transforms $\overline{\mathcal{P}}_{i}$ vanish at the boundaries $z= \pm L$ of the confinement regions that extend on either side of the ridge. Because the antiproton distribution is an even function of the height $z$, the interested reader may also show that $\dot{\mathcal{P}}_{i}\left(0_{+}\right)=-\dot{\overline{\mathcal{P}}}_{i}\left(0_{-}\right)=h \Gamma_{\bar{p}} \overline{\mathcal{P}}_{i}(0) / K$. The thin 
disk approximation implies a discontinuity of the derivative $\overline{\mathcal{P}}_{i}$ for $z=0$. The final result is readily obtained as

$$
\overline{\mathcal{P}}_{i}(z>0)=\frac{2}{K S_{i}}\left\{\mathcal{F}(L) \frac{\mathcal{G}(z)}{\mathcal{G}(L)}-\mathcal{F}(z)\right\} .
$$

This expression describes the actual propagation of antiprotons which have been produced in remote regions of the halo and that propagate backwards in the magnetic fields of the Galaxy. The functions $\mathcal{F}(z)$ and $\mathcal{G}(z)$ are respectively defined by

$$
\mathcal{F}(z>0)=\int_{0}^{z} \sinh \left\{\frac{S_{i}}{2}\left(z-z^{\prime}\right)\right\} q_{i}^{\mathrm{SUSY}}\left(z^{\prime}\right) d z^{\prime}
$$

and

$$
\mathcal{G}(z>0)=2 h \Gamma_{\bar{p}} \sinh \left(\frac{S_{i} z}{2}\right)+K S_{i} \cosh \left(\frac{S_{i} z}{2}\right) .
$$

The interstellar flux at the solar system of the antiprotons produced by the annihilation of hypothetical supersymmetric species comprising part of the galactic halo may now be expressed as

$$
\Phi_{\bar{p}}^{-}\left(\odot, T_{\bar{p}}^{-}\right)=\left\langle\sigma_{\mathrm{ann}} v\right\rangle g\left(T_{\bar{p}}^{-}\right)\left\{\frac{\rho_{0}}{m_{\chi}}\right\}^{2} C_{\mathrm{SUSY}}\left(T_{\bar{p}}^{-}, f\right)
$$

The density of reference $\rho_{0}$ has been set equal to $1 \mathrm{GeV} \mathrm{cm}^{-3}$. The coefficient $C_{\mathrm{SUSY}}\left(T_{\bar{p}}, f\right)$ is defined as

$$
C_{\mathrm{SUSY}}\left(T_{\bar{p}}^{-}, f\right)=\frac{1}{4 \pi} v_{\bar{p}}^{-} \psi_{\bar{p}}^{\mathrm{eff}}\left(\odot, T_{\bar{p}}^{-}\right)
$$

The effective energy distribution $\psi_{\bar{p}}^{\text {eff }}$ is taken at the solar circle and has been derived with the abovementioned method where an effective antiproton source term $\left\{\rho_{\chi}(r, z) / \rho_{0}\right\}^{2}$ has been assumed. The latter term depends on the flattening $f$ of the halo. Note that $C_{\text {SUSY }}$ is not a flux of particles. It is a mere coefficient that is actually expressed in units of $\mathrm{cm} \mathrm{sr}^{-1}$. Figure 6 illustrates the behavior of this coefficient when the antiproton kinetic energy is varied from $100 \mathrm{MeV}$ up to $10 \mathrm{GeV}$, for three different values of the flattening factor $f=0.1,0.5$, and 1 . The coefficient $C_{\mathrm{SUSY}}\left(T_{p}^{-}, f\right)$ exhibits a smooth maximum around $T_{p}^{-} \sim 1 \mathrm{GeV}$. Below that value, it tends to decrease with the antiproton velocity like $v_{p}^{-} / B_{i}$. For higher energies, the diffusion takes place more efficiently and the cosmic rays escape more easily from the galactic magnetic fields, hence a lower density in the disk. When the flattening increases, the dark matter halo is compressed towards the ridge. There are many more neutralinos in the diffusion layers where antiprotons are kept confined, hence a larger flux. The evaluation of the IS $\bar{p}$ flux due to neutralino annihilation is then performed by using Eq. (45).

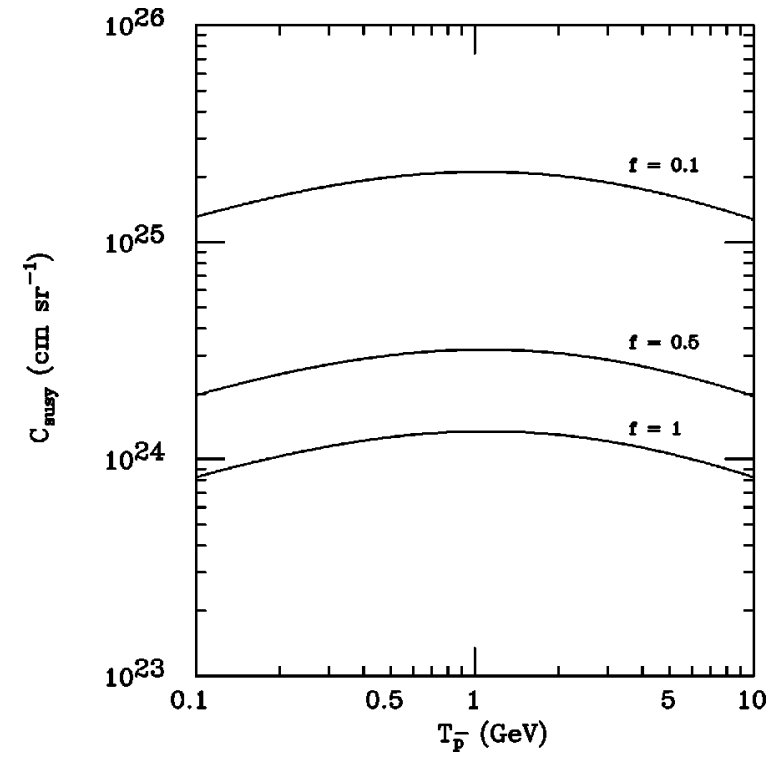

FIG. 6. Coefficient $C_{\mathrm{SUSY}}\left(T_{\bar{p}}^{-}, f\right)$ as a function of the $\bar{p}$ kinetic energy for different values of the flattening parameter $f$.

\section{PRIMARY AND SECONDARY ANTIPROTON TOA FLUXES}

Our TOA antiproton fluxes are derived from the corresponding IS spectra, by employing the Perko solarmodulation procedure [21], already defined in Sec. II. In that section we also derived the values for the parameter $\Delta$ relevant to the measurements of Refs. $[19,20]$. In order to obtain the $\Delta$ values to be applied in case of experiments performed at different times, we use the results of Papini, Grimani, and Stephens (PGS) [24]. These authors derived simple analytic expressions as best fits to the measured spectra of the TOA primary cosmic-ray protons, obtained from a large collection of data over a couple of solar cycles. They provide the parameters of these fits for periods of maximum and minimum solar activity. By fitting their analytic expressions with the solar-modulated flux derived from our parametric form of Eq. (1), we find the following average values for $\Delta$ at minima and maxima: $\Delta_{\min }=320 \mathrm{MeV}$ and $\Delta_{\max }$ $=800 \mathrm{MeV}$, respectively. When Eq. (2) is used, the values $\Delta_{\min }=560 \mathrm{MeV}$ and $\Delta_{\max }=1010 \mathrm{MeV}$ are obtained.

In Fig. 7 we plot the time variation of the solarmodulation parameter $\Delta$, as obtained by our best fit to the experimental data by employing the parametrization of Eq. (1). The full circles represent the best-fit values to the PGS average fluxes at minima $(\min )$ and at maxima $(\max )$, and to the fluxes of Refs. [19,20]. The open circle refers to the BESS95 data taking period. The cross denotes the extrapolated value at the time relevant for the AMS measurements with the Shuttle flight.

In Figs. 8 and 9 we display how the effects of the flux distortion at low energies, induced by solar modulation, is much stronger for the primary flux than for the secondary one. This may be simply understood in terms of the nature of Eq. (3) and of the different shapes for secondaries and primaries. In turn, this implies that the primary fluxes are more sizeably affected than the secondary fluxes by the choice for 


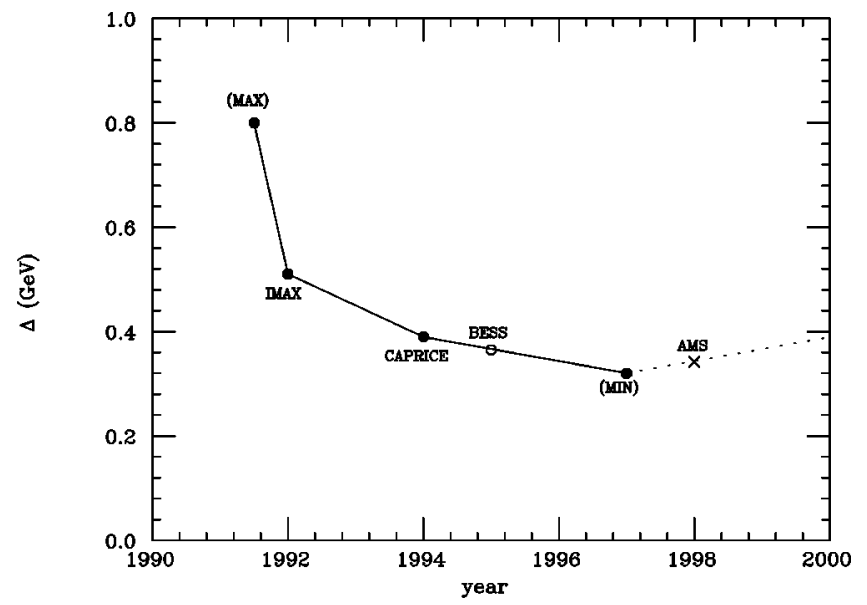

FIG. 7. Time variation of the solar-modulation parameter $\Delta$ as obtained from parametrization of Eq. (1). Full circles represent the best-fit values to the PGS average fluxes at minima (min) and at maxima $(\max )$ and to the fluxes of IMAX [19] and of CAPRICE [20]; the open circle refers to the BESS95 data taking period and the cross denotes the extrapolated value of $\Delta$ at the time relevant for the future AMS Shuttle flight (May 1998).

the parametrization of the IS proton flux. As already mentioned, in the present paper we thoroughly pursue our analysis using parametrization of Eq. (1). However, some comments on how the physical conclusions of our paper would modify, if Eq. (2) is used instead of Eq. (1), are added in Sec. VI.

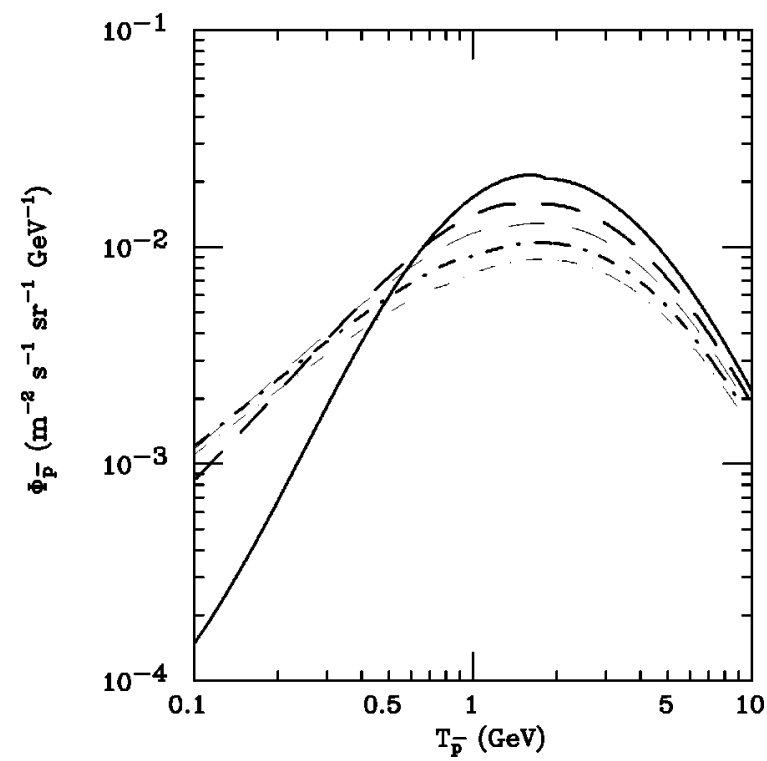

FIG. 8. Solar modulation of the IS median secondary antiproton flux calculated in this paper. Solid line is the IS spectrum. The thick dashed (thick dot-dashed) line is the solar-modulated spectrum at minima (maxima) when the modulation parameter $\Delta$ is obtained from parametrization of Eq. (1) for the primary proton spectrum. The light dashed (light dot-dashed) line is the solar-modulated spectrum at minima (maxima) when the modulation parameter $\Delta$ is obtained from parametrization of Eq. (2).

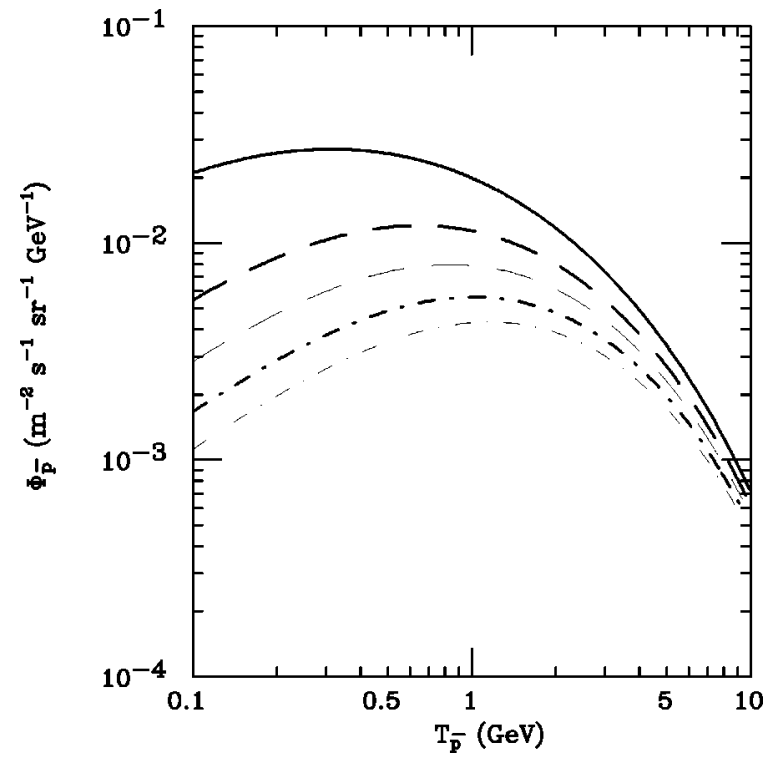

FIG. 9. Solar modulation of the IS antiproton flux, due to neutralino annihilation for the representative neutralino configuration with $m_{\chi}=62 \mathrm{GeV}, P=0.98$, and $\Omega_{\chi} h^{2}=0.11$. The solid line is the IS spectrum. The thick dashed (thick dot-dashed) line is the solarmodulated spectrum at minima (maxima) when the modulation parameter $\Delta$ is obtained from parametrization of Eq. (1) for the primary proton spectrum. The light dashed (light dot-dashed) line is the solar-modulated spectrum at minima (maxima) when the modulation parameter $\Delta$ is obtained from parametrization of Eq. (2).

\section{COMPARISON WITH EXPERIMENTAL DATA}

Early measurements of cosmic-ray antiprotons have been plagued by low-statistics problems and brought to serious conflicting results at low energies $\left(T_{\bar{p}} \lesssim 0.4 \mathrm{GeV}\right)$ in the past [45]. As already mentioned in our Introduction, a recent analysis [1] of the data collected by the BESS spectrometer during its 1995 flight (BESS95) has provided a significant improvement in statistics in the low-energy region, with a total of $43 \bar{p}$ 's in the kinetic-energy range $180 \mathrm{MeV} \leqslant T_{\bar{p}}$ $\leqslant 1.4 \mathrm{GeV}$ [46]. This then allows an interpretation of the experimental data in terms of theoretical models in a more meaningful way than in the past. A further substantial breakthrough in this direction will be provided by the forthcoming measurements with AMS [15], the satellite-borne PAMELA experiment [16], and balloon-borne measurements [17].

The BESS95 data are displayed in Fig. 1 and compared with our theoretical evaluations for secondary antiprotons. Our curves are derived according to the procedure outlined in previous sections. Solar modulation is evaluated at the time of the BESS95 measurement. The band delimited by the dotted and the dashed curves provides the uncertainty in the secondary $\bar{p}$ 's flux due to the corresponding uncertainty in the primary IS cosmic ray proton flux (see Sec. II). It turns out that this uncertainty is $\leqslant \pm 30 \%$ for $T_{p}^{-} \leqslant 2 \mathrm{GeV}$ and it grows up to $\pm 50 \%$ at $T_{p}^{-} \simeq 10 \mathrm{GeV}$.

From a first look at Fig. 1 it is apparent that the experimental data are consistent with the flux due to secondary $\bar{p}$ 's. This is indeed quantitatively confirmed by a $\chi^{2}$ evaluation, which shows that our median curve for secondaries fits the 
BESS95 data with a low reduced- $\chi^{2}$ value $\left(\chi^{2}\right)_{\text {red }}=0.83$ (for $5 N_{\mathrm{DF}}$ ) [47].

However, it is interesting to explore which would be the chances for a signal, due to relic neutralino annihilations, of showing up in the low-energy window $\left(T_{p}^{-} \lesssim 1 \mathrm{GeV}\right)$. This point is very challenging, especially in view of the interplay which might occur among low-energy measurements of cosmic-ray $\bar{p}$ 's and other searches, of quite a different nature, for relic neutralinos in our Galaxy.

Actually, we find that the agreement between BESS95 experimental data and theory may be improved by adding a fraction of neutralino-induced $\bar{p}$ 's to the standard secondary antiprotons. The best fit to the experimental data with a total theoretical flux $\Phi^{\text {th }}=\Phi_{\text {med }}^{\text {sec }}+\Phi^{\text {SUSY }}$, performed by varying the supersymmetric parameters over the grid defined in Sec. II B, provides a value $\left(\chi^{2}\right)_{\text {red }}=0.28$, with an improvement over the $\left(\chi^{2}\right)_{\text {red }}$ previously obtained by using the secondary flux only. This fact certainly cannot be taken as significant evidence of a neutralino-induced antiproton signal, but shows that indeed the low-energy region $\bar{p}$ spectrum is still a quite interesting window for exploring $\bar{p}$ 's of supersymmetric origin, and encourages further investigation of the problem.

Now we wish to specifically determine which regions of the supersymmetric parameter space (and then which neutralino configurations) might be relevant for the problem at hand and how these could be investigated by other experimental means. As a quantitative criterion to select the relevant supersymmetric configurations, we choose to pick up only the configurations which meet the following requirements: (i) they generate a total theoretical flux $\Phi^{\text {th }}$ which is at least at the level of the experimental value (within 1- $\sigma$ ) in the first energy bin; (ii) their $\left(\chi^{2}\right)_{\text {red }}$, in the best fit of the BESS95 data, is bounded by $\left(\chi^{2}\right)_{\text {red }} \leqslant 2.2$ (corresponding to 95\% C.L. for $5 N_{\mathrm{DF}}$ ). This set of configurations is hereafter denoted as set $M$; its subset, whose $\Omega_{\chi} h^{2}$ values fall in the cosmologically interesting range $0.03 \leqslant \Omega_{\chi} h^{2} \leqslant 0.7$, is denoted as set $N$. An example of a fit to the BESS95 data which includes a neutralino-induced signal with a $\left(\chi^{2}\right)_{\text {red }}$ $\leqslant 2.2$ is shown in Fig. 10. This signal corresponds to a neutralino with the following properties: $m_{\chi}=62 \mathrm{GeV}, P$ $=0.98$, and $\Omega_{\chi} h^{2}=0.11$.

On the other hand, supersymmetric configurations with a $\left(\chi^{2}\right)_{\text {red }}>4$ have to be considered as strongly disfavored by BESS95 data (actually, they are excluded at 99.9\% C.L.). We call $R$ this set of supersymmetric configurations and we will discuss them later on. Supersymmetric configurations belonging neither to $M$ nor to $R$ can only provide a $\bar{p}$ flux fully buried in the secondary $\bar{p}$ flux and are then completely irrelevant for the problem under discussion.

The composition of configurations in sets $M$ and $N$ are displayed in Fig. 11 (Fig. 12), where $\tan \beta\left(m_{\chi}\right)$ is plotted in terms of the fractional amount of gaugino fields, $P=a_{1}^{2}+a_{2}^{2}$, in the neutralino mass eigenstate. From Fig. 11 we notice that gaugino configurations are more numerous than others, with only a slight correlation with $\tan \beta$; the requirement of a sizeable contribution to the relic density

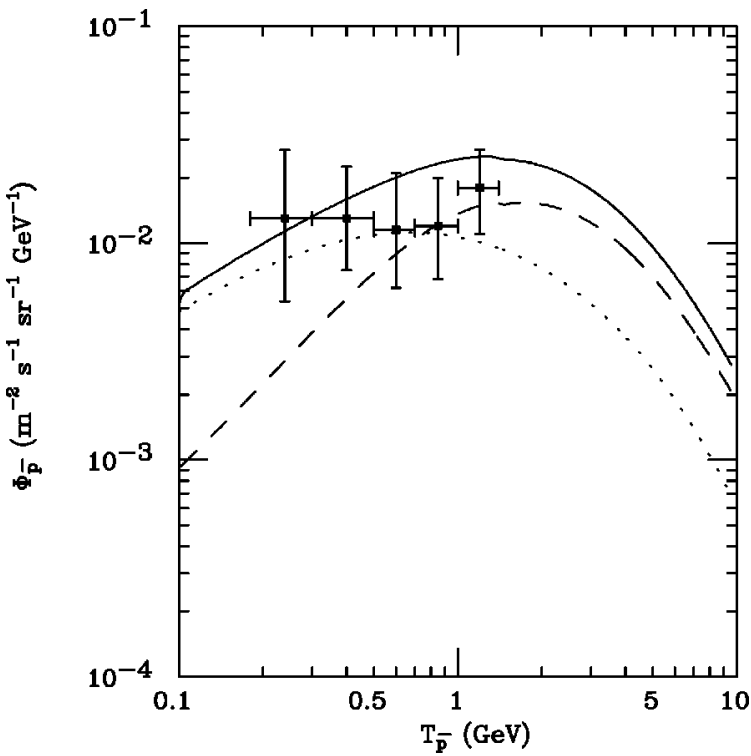

FIG. 10. TOA antiproton fluxes versus the antiproton kinetic energy. The BESS95 data [1] are shown by crosses. The dashed line denotes the median secondary flux, the dotted one denotes the primary flux due to neutralino annihilation in the halo for a neutralino configuration with $m_{\chi}=62 \mathrm{GeV}, P=0.98$, and $\Omega_{\chi} h^{2}=0.11$. The solid line denotes the calculated total flux.

introduces a noticeable reduction in the number of Higgsinolike and mixed configurations. Figure 12 shows that Higgsino-like and mixed configurations are much stronger constrained in the neutralino mass range than the gauginolike ones. In Fig. 13 we display the features of configurations of set $R$. These configurations, which are to be considered excluded on the basis of the BESS95 data, turn out to be gauginolike with masses on the low side.
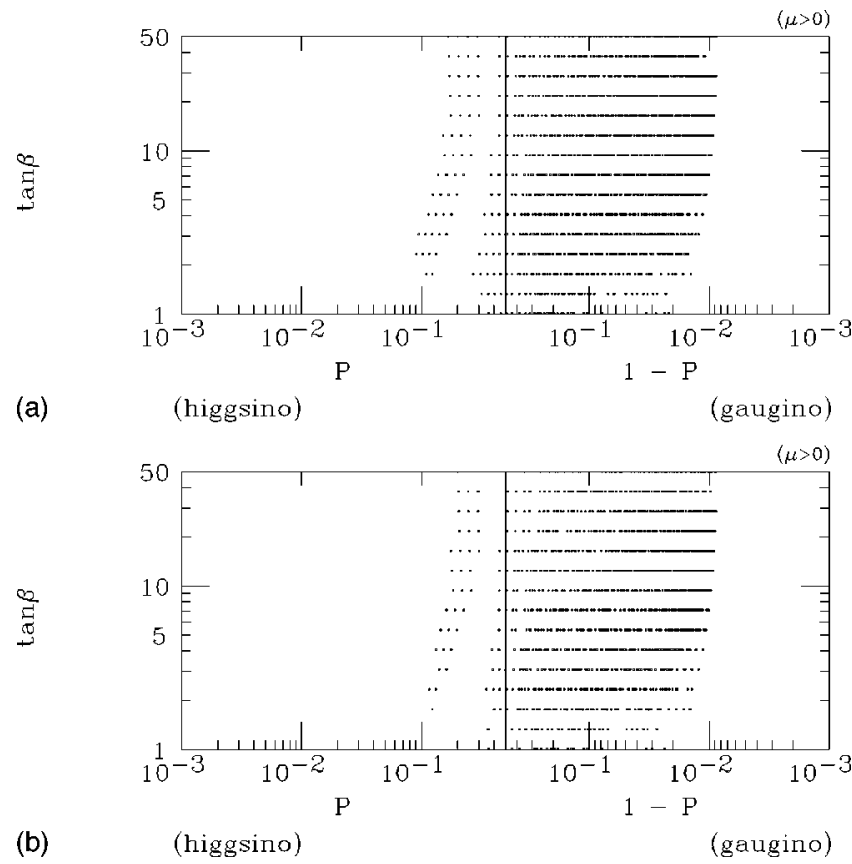

FIG. 11. Scatter plots for configurations of set $M$ (a) and set $N$ (b) in the $P-\tan \beta$ plane. 


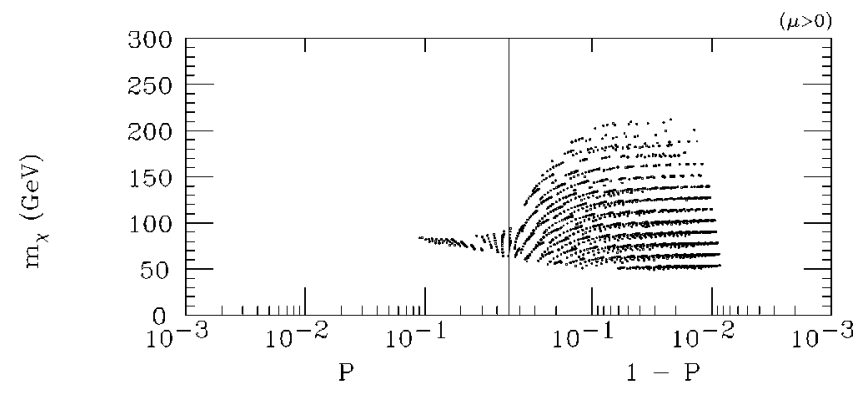

(a)

(higgsino)

(gaugino)

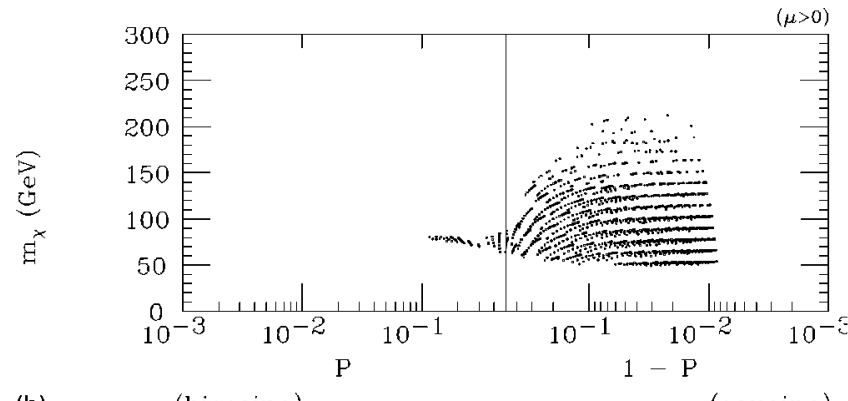

(b)

(higgsino)

(gaugino)

FIG. 12. Scatter plots for configurations of set $M$ (a) and set $N$ (b) in the P- $m_{\chi}$ plane.

It is clear that the specific features of the SUSY configurations belonging to the various sets: $M, N, R$ depend on a number of assumptions we have taken from the very beginning. One of the relevant assumptions concerns the size of the solar modulation effect on the primary antiproton flux. For instance, as we have shown above, the use of parametrization of Eq. (2) for the primary IS proton flux would imply
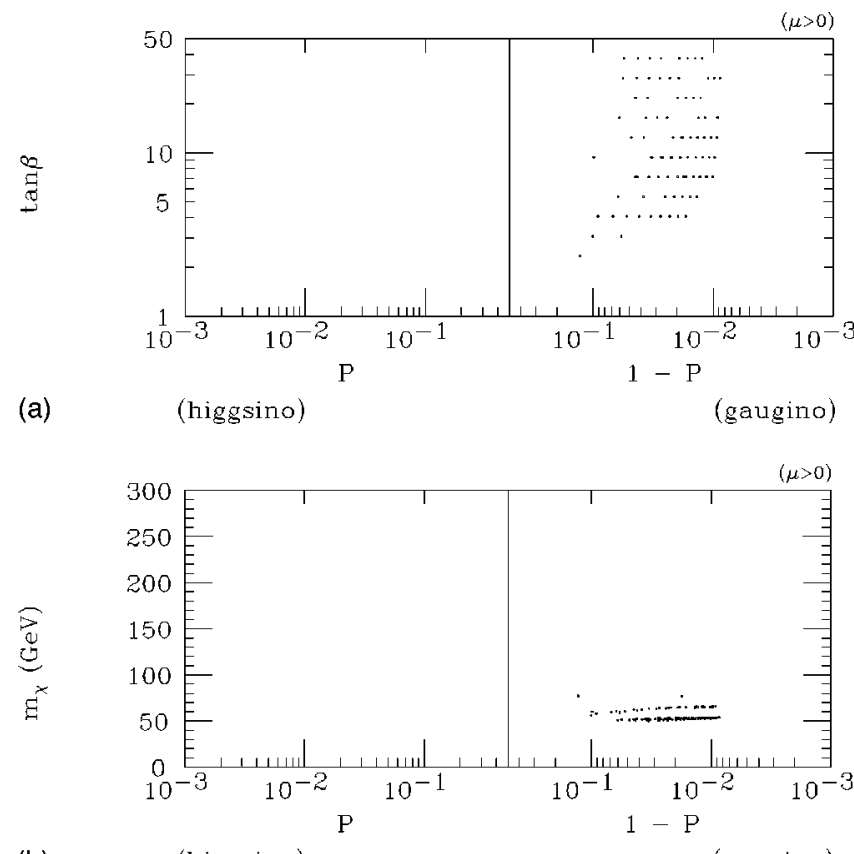

(b)

(higgsino)

(gaugino)

FIG. 13. Scatter plots for configurations of set $R$ in the $P-\tan \beta$ plane (a) and in the $P-m_{\chi}$ plane (b).

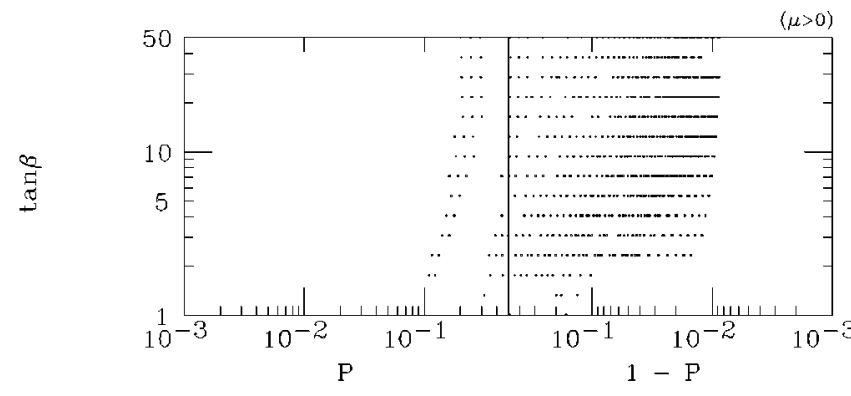

(a)

(higgsino)

(gaugino)

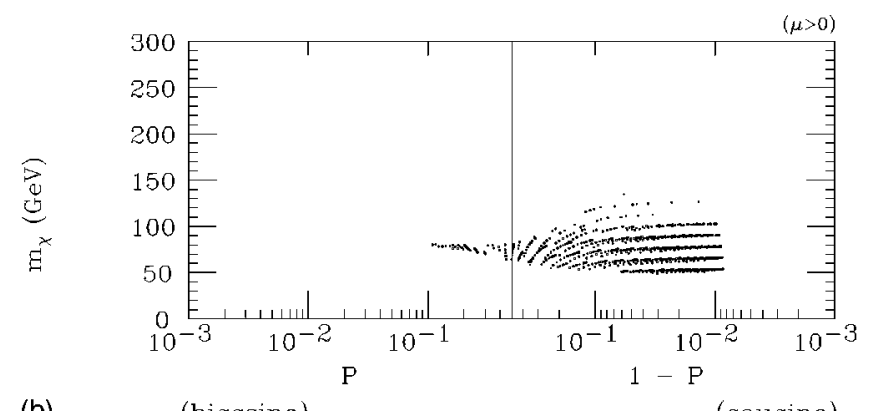

(b)

(higgsino)

(gaugino)

FIG. 14. Scatter plots for configurations of set $R$ in the $P$-tan $\beta$ plane (a) and in the $P-m_{\chi}$ plane (b) for a flattening of $f=0.5$.

a stronger solar modulation effect, and consequently a reduction of antiproton primary signals. In turn, most of the supersymmetric configurations previously included in set $R$, would now play the role of relevant configurations for a good fit of the BESS95 data (i.e., they would belong now to set $M$ ). Simultaneously some configurations, previously belonging to set $M$, would become irrelevant; this would happen mainly to those of higher neutralino masses and of Higgsino dominance.

Up to now, we have discussed our results in terms of a spherically symmetric galactic halo. The effect of a flattening in the dark matter distribution is to enhance the primary $\bar{p}$ flux. Since the size of this flux is proportional to the function $C_{\text {SUSY }}\left(T_{\bar{p}}, f\right)$, defined in Sec. IV, the enhancement of the primary flux as a function of $f$ may be read directly from Fig. 6. For instance, for $f=0.5$ the enhancement factor is 2.3 . This has consequences on the nature of configurations in sets $M, N$, and $R$. By way of example, we plot in Fig. 14 the scatter plot for configurations of set $R$ for a flattening of $f$ $=0.5$. This may be compared with the corresponding plots of Fig. 13 which refer to $f=1$. Obviously, the enhancement of the primary flux, induced by the halo flattening, increases the number of excluded configurations.

In the present paper we have considered only uniform dark matter distribution inside the density profile of Eq. (10). As is well known, any effect of local density enhancement or clumpiness would induce a substantial increase in the primary $\bar{p}$ spectrum, as in any other signal due to pair annihilation taking place in the halo [48]. Let us now examine whether our relevant neutralino configurations may be explored in terms of direct detection experiments for particle dark matter candidates. 

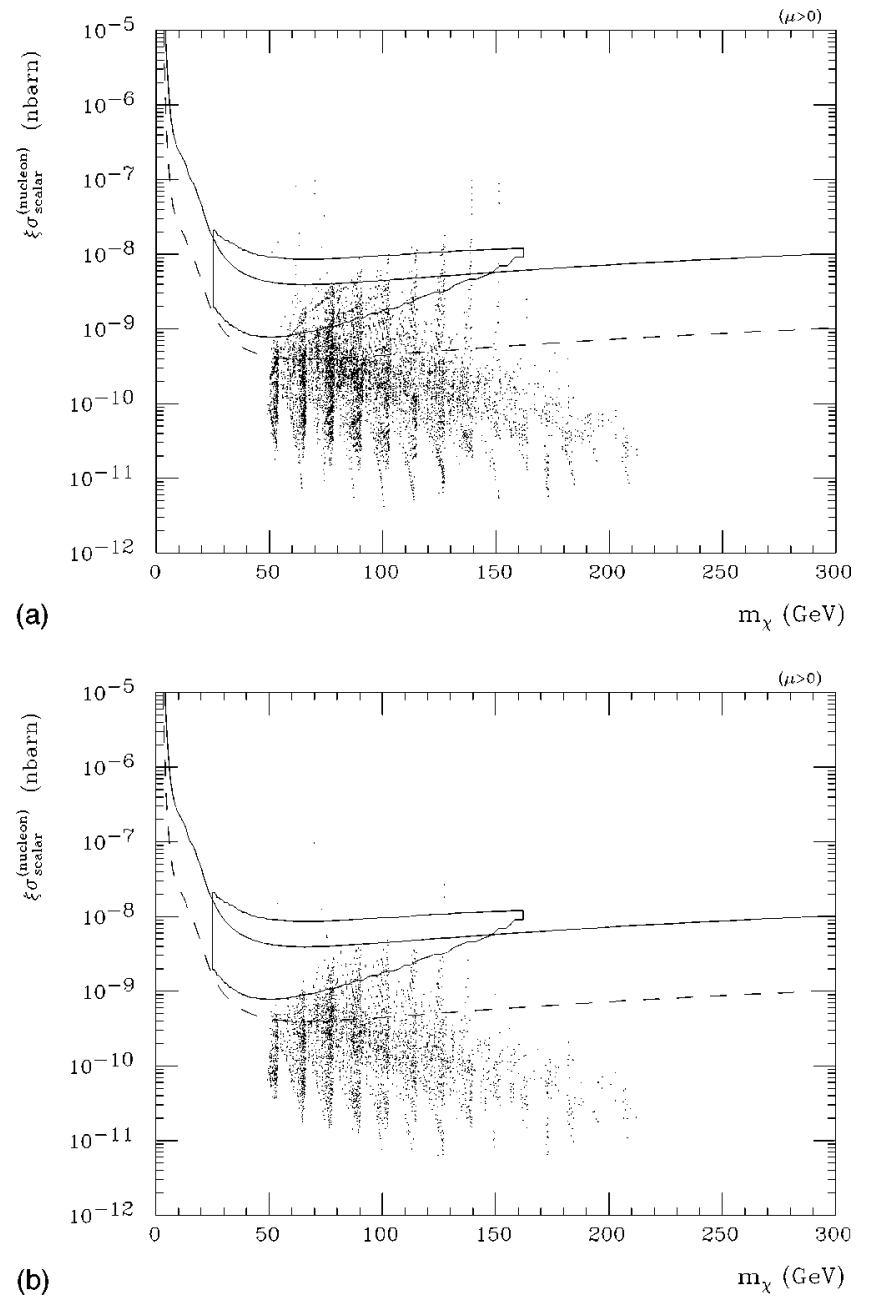

FIG. 15. Scatter plot of the values of $\xi \sigma_{\text {scalar }}^{\text {nuclen }}$ versus the neutralino mass for the configurations of set $M$ (a) and of set $N$ (b). The open curve denotes the (90\% C.L.) upper bound obtained from experimental data of Ref. [51]. The region delimited by a closed contour is the one singled out by the experiment of Ref. [52] as possibly indicative of an annual modulation effect. The total local dark matter density is normalized here to the value $\rho_{l}$ $=0.4 \mathrm{GeV} \mathrm{cm}^{-3}$. The dashed line shows the discovery potential in case of an improvement by a factor of 10 in current sensitivities for experiments of direct search for particle dark matter.

\section{EXPLORATION BY DIRECT DETECTION OF RELIC PARTICLES}

The measurements of the energy differential rates in experiments of direct search for particle dark matter enable the extraction of an upper bound for the neutralino-nucleon scalar cross section $\sigma_{\text {scalar }}^{\text {nucleon }}$, multiplied by the neutralino local (solar neighborhood) density, i.e., an upper bound for the quantity $\xi \sigma_{\text {scalar }}^{\text {nuclen }}$, once a specific value to the total local dark matter density is assigned [49]. By combining all present experimental data [50], we obtain the (90\% C.L.) upper bound displayed in Fig. 15 by the open solid curve (the total local dark matter density is normalized here and in the rest of this paper to the value $\rho_{l}=0.4 \mathrm{GeV} \mathrm{cm}^{-3}$ ). The experiments which are essential in the determination of this

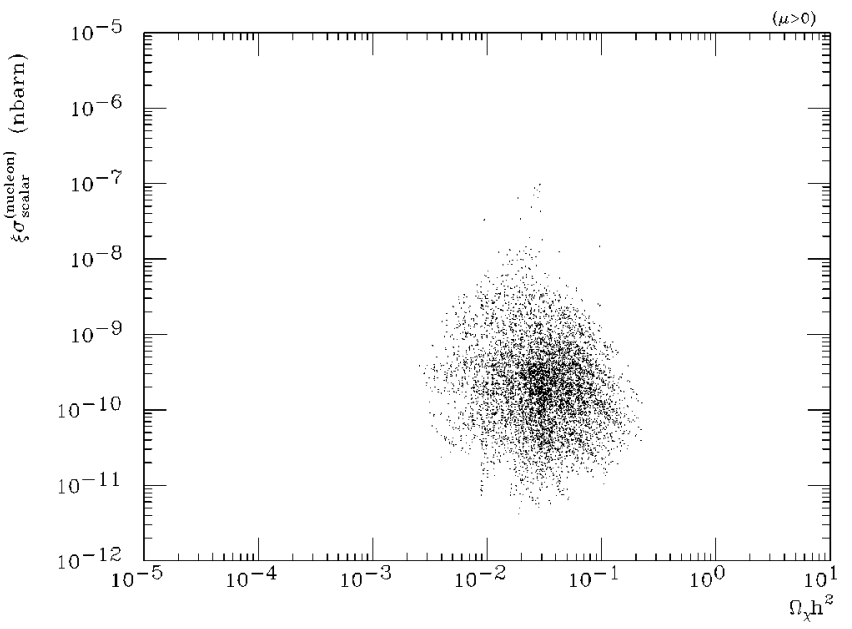

FIG. 16. Correlation between $\xi \sigma_{\text {scalar }}^{\text {nuclen }}$ and the neutralino relic density $\Omega_{\chi} h^{2}$ for configurations of set $M$.

upper bound, in the neutralino mass range considered here, are those of Refs. [51]. The region in Fig. 15 delimited by a closed contour is the one singled out by the experiment of Ref. [52] as possibly indicative of an annual modulation effect (for an interpretation of these data in terms of relic neutralinos see Ref. [53]). The scatter plot displays the values of $\xi \sigma_{\text {scalar }}^{\text {nucleon }}$ for the configurations of set $M$ [part (a) of Fig. 15] and of set $N$ [part (b)]. It is most remarkable that a sizeable fraction of the configurations are accessible to investigation by direct detection, since the sensitivity in this kind of experiments is expected to be significantly improved in the near future [50]. The dashed line in Fig. 15 shows the discovery potential in case of an improvement by a factor of 10 in current sensitivities, what is within reach in a short time. Our analysis shows an interesting interplay between experiments of direct search for particle dark matter and measurements of low-energy $\bar{p}$ 's in space. This property would obviously be dramatically reinforced, should the indication about a possible annual modulation effect be confirmed by new data. In fact, it is very intriguing that many configurations of set $M$ are indeed in the region singled out by the experiment of Ref. [52]. Finally, we notice that some configurations are actually excluded by the direct-search upper bound. This put emphasis on the potentiality of direct detection measurements in providing information on dark matter searches of different nature.

Part (b) of Fig. 15 shows how the requirement of a sizeable contribution to the relic abundance makes somewhat thinner the set of configurations contributing to the highest values of $\xi \sigma_{\text {scalar }}^{\text {nuclen }}$, but still leaves a significant number of configurations inside the closed region and, anyway, close to the current upper bound curve. Correlation between $\xi \sigma_{\text {scalar }}^{\text {nucleon }}$ and the neutralino relic density is given in Fig. 16.

\section{SEARCH AT ACCELERATORS}

Let us turn now to the question of whether configurations of sets $M$ and $N$ might be explored at accelerators. LEP at $\sqrt{s}=192 \mathrm{GeV}$ may explore the configurations with a neu- 


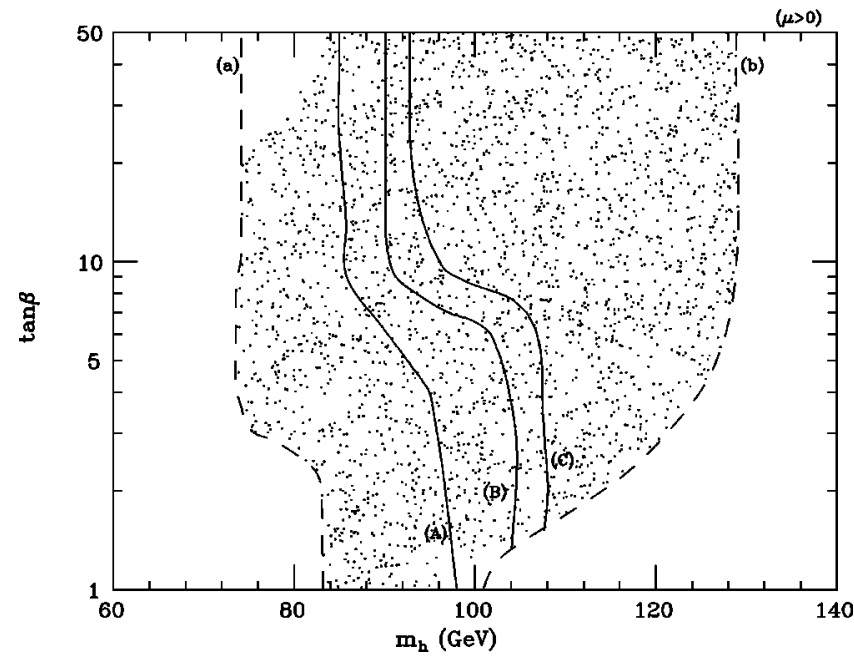

FIG. 17. Scatter plot for configurations of set $M$ in the $m_{h}$-tan $\beta$ plane. The region on the left of the dashed line denoted by (a) is excluded by current LEP experimental data [34], the one on the right of the dashed line (b) is theoretically disallowed. The other lines display the LEP reach at luminosity $L=200 \mathrm{pb}^{-1}$ and various energies [54]: (A) discovery potential at $\sqrt{s}=192 \mathrm{GeV}$; (B) discovery potential at $\sqrt{s}=200 \mathrm{GeV}$; (C) exclusion at $\sqrt{s}$ $=200 \mathrm{GeV}$.

tralino mass up to $\simeq 50 \mathrm{GeV}$ [54]. Then, from Fig. 12 we see that LEP will be able to investigate only marginally the configurations of set $M$ and $N$ in the gaugino sector. Experimental investigation of larger masses requires future upgrading of the Tevatron or LHC. For instance, TeV33 could, under favorable hypothesis, explore a range up to $m_{\chi}$ $\simeq 125 \mathrm{GeV}$ [55]. In this case, all the Higgsino configurations can be explored, as well as a large portion of the gaugino sector.

A further illustrative point is offered by a scatter plot of set $M$ in the plane $m_{h}-\tan \beta$, displayed in Fig. $17\left(m_{h}\right.$ is the mass of the lightest $C P$-even scalar Higgs boson). The representative points of the set cover almost completely the Higgs physical region. Part of these supersymmetric configurations (the ones on the left side of the solid curves) will be explored by LEP at $\sqrt{s}=192 \mathrm{GeV}$ and at $\sqrt{s}=200 \mathrm{GeV}$, with a luminosity of $200 \mathrm{pb}^{-1}$ per experiment [54].

\section{CONCLUSIONS}

We have presented a new analysis of the cosmic-ray antiprotons flux, expected on the basis of secondary $\bar{p}$ 's, generated by interactions of cosmic-ray primaries with the interstellar medium, and of a possible exotic primary source of $\bar{p}$ 's, originated by neutralino-neutralino annihilations in the Galactic halo.

Improvements over previous calculations of secondaries depend mostly on (i) the use of a two-zone propagation model for diffusion of cosmic rays in the halo instead of the standard leaky box model; (ii) the inclusion of an energy-loss effect in the propagation properties of cosmic rays (important for the antiproton low-energy range considered in this paper); (iii) the use of the new data on primary cosmic-ray

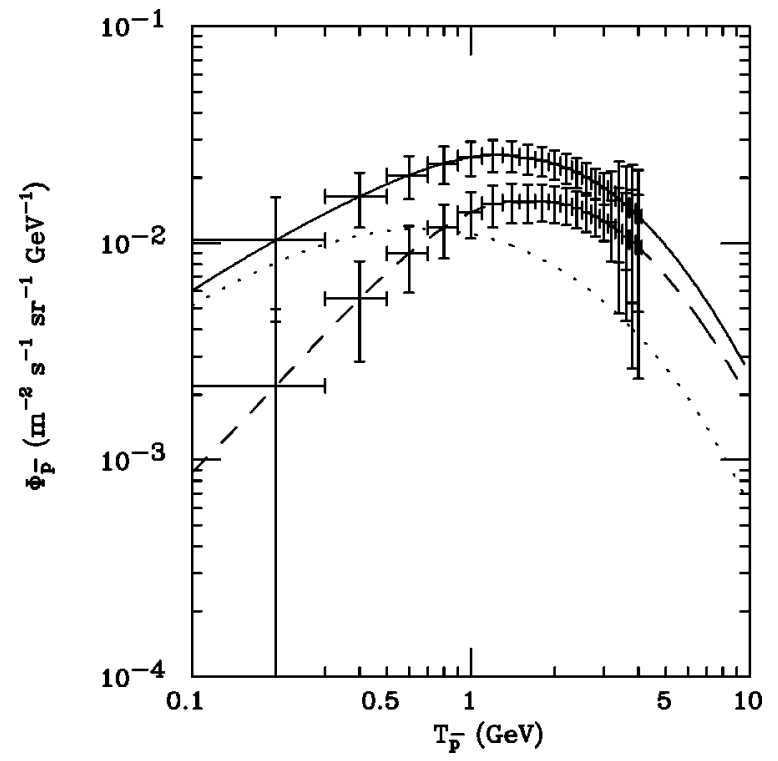

FIG. 18. Expected distribution of measurements with the AMS Shuttle flight according to two different hypotheses: (a) dominance of the secondary contribution (lower sequence of crosses), (b) significant contribution due a neutralino-induced signal (upper sequence of crosses). The dashed line denotes the secondary flux, the dotted one denotes the primary flux due to neutralino annihilation in the halo for a neutralino configuration with the representative values $m_{\chi}=62 \mathrm{GeV}, P=0.98$, and $\Omega_{\chi} h^{2}=0.11$. The solid line denotes the calculated total flux.

proton spectrum, as measured by IMAX [19] and CAPRICE [20].

The neutralino-induced $\bar{p}$ flux has been evaluated in a MSSM at the electroweak scale, which incorporates all current accelerator constraints. Use of supergravity-inspired unification conditions at large energy scale has been avoided in order not to arbitrarily constrain the neutralino phenomenology [56]. Solar modulation of the antiproton flux has been improved by analyzing the most complete set of data over the solar cycles [24] and the data on the proton spectrum of Refs. [19,20].

We have found that the most statistically relevant data on cosmic-ray antiprotons at low energy [1] leave some room for a possible signal from neutralino annihilation in the galactic halo. We have discussed how the relevant supersymmetric configurations may be explored with direct experiments for particle dark matter search and at accelerators. We have shown how the interplay between measurements of cosmic-ray $\bar{p}$ 's and direct search experiments for relic particles is very intriguing and quite important in view of the significant improvements expected in these two classes of experiments in the near future. The present analysis stresses the great interest in the forthcoming AMS measurements with the Shuttle flight and on the ISSA [15], as well as for other future measurements with balloon-borne experiments (IMAX [4], BESS [5]) and with satellites (PAMELA) [16], for disentangling the secondary $\bar{p}$ flux from a possible primary signal of exotic nature. As an example, we give in Fig. 18 the distribution of measurements expected for AMS with 
the Shuttle flight according to two different hypotheses: (a) dominance of the secondary contribution (lower sequence of crosses), (b) significant contribution due a neutralinoinduced signal (upper sequence of crosses). In our evaluation of the expected measurements we have taken into account geomagnetic cutoff effects and the expected AMS overall acceptance [57]. A word of caution, however, one should keep in mind that the estimate of the secondary antiproton yield suffers from uncertainties of various origins. The difficulty to measure accurately the proton flux itself translates into a shift on the antiproton signal by $\pm 25 \%$. The diffusion coefficient adds up a mere $\pm 45 \%$ at low energies $(100 \mathrm{MeV})$ and $\pm 20 \%$ at larger ones (above $0.6 \mathrm{GeV}$ ).

\section{ACKNOWLEDGMENTS}

We thank Roberto Battiston and Aldo Morselli for interesting discussions about experimental aspects related to the present paper. P.S. would like to thank the French Programme National de Cosmologie as well as the French Groupement de Recherche de Supersymétrie for their support. A.B. and N.F. acknowledge Research Funds of the Ministero dell'Università e della Ricerca Scientifica e Tecnologica.
[1] BESS Collaboration, H. Matsunaga et al., in Proceedings of the 25th International Conference of Cosmic Rays, Durban, 1997, edited by M. S. Potgieter, B. C. Raubenheimer, and D. J. van der Walt (World Scientific, Singapore, 1998).

[2] R.L. Golden et al., Phys. Rev. Lett. 43, 1196 (1979); E.A. Bogomolov et al., in Proceedings of the 16th International Conference of Cosmic Rays, Kyoto, 1979, edited by S. Hiyake (Institute for Cosmic Ray Research, Tokyo, 1979); A. Buffington, S.M. Schindler, and C. Pennypacker, Astrophys. J. 248, 1179 (1981); A. Buffington and S.M. Schindler, Astrophys. J., Lett. Ed. 248, L105 (1981); PBAR Collaboration, S.P. Ahlen et al., Phys. Rev. Lett. 61, 145 (1988); R.E. Streitmatter et al., Adv. Space Res. 9, 1265 (1989).

[3] MASS2 Collaboration, M. Hof et al., in Proceedings of the 24th International Conference of Cosmic Rays, Rome, 1995, edited by A. Taroni (Ed. Compositori, Bologna, 1997) [Nuovo Cimento C 19, 623 (1997)]; M. Hof et al., Astrophys. J. Lett. 467, L33 (1996).

[4] IMAX Collaboration, J.W. Mitchell et al., Phys. Rev. Lett. 76, 3057 (1996).

[5] BESS Collaboration, A. Moiseev et al., Astrophys. J. 474, 479 (1997)

[6] CAPRICE Collaboration, G. Barbiellini et al., in Proceedings of the 25th International Conference of Cosmic Rays, Durban, 1997 [1]; M. Boezio et al., Astrophys. J. 487, 415 (1997).

[7] J. Silk and M. Srednicki, Phys. Rev. Lett. 53, 624 (1984); J. Ellis, R.A. Flores, K. Freese, S. Ritz, D. Seckel, and J. Silk, Phys. Lett. B 214, 403 (1988); F. Stecker, S. Rudaz, and T. Walsch, Phys. Rev. Lett. 55, 2622 (1985); J.S. Hagelin and G.L. Kane, Nucl. Phys. B263, 399 (1986); S. Rudaz and F.W. Stecker, Astrophys. J. 325, 16 (1988); F. Stecker and A. Tylka, Astrophys. J. Lett. 336, L51 (1989); G. Jungman and M. Kamionkowski, Phys. Rev. D 49, 2316 (1994).

[8] A. Bottino, C. Favero, N. Fornengo, and G. Mignola, Astropart. Phys. 3, 77 (1995).

[9] T. Mitsui, K. Maki, and S. Orito, Phys. Lett. B 389, 169 (1996).

[10] P. Kiraly, J. Szabelski, J. Wdwczyk, and A.W. Wolfendale, Nature (London) 293, 120 (1981); M.S. Turner, ibid. 297, 379 (1982); J.H. MacGibbon and B.J. Carr, Astrophys. J. 371, 447 (1991); K. Maki, T. Mitsui, and S. Orito, Phys. Rev. Lett. 76, 3474 (1996).
[11] E. Witten, Nucl. Phys. B249, 557 (1985); G.D. Starkman and T. Vachaspati, Phys. Rev. D 53, R6711 (1996).

[12] See, for instance, T.K. Gaisser, Cosmic Rays and Particle Physics (Cambridge University Press, Cambridge, England, 1992).

[13] A possible interpretation of BESS95 data in terms of neutralino annihilation is also suggested in Ref. [1]. However, the theoretical models used in that analysis (taken from Ref. [9]) differ from ours both for primary and secondary fluxes. In particular, the supersymmetric model employed there is a minimal $N=1$ supergravity model, at variance with the one used in the present paper (MSSM at the electroweak scale). Furthermore, a large enhancement effect in the primary flux (induced by clumpiness in the halo) is introduced in that analysis.

[14] Preliminary results of this analysis were presented in talks delivered at the International Workshops Tools for SUSY, Annecy, 1998 (unpublished); and Matter, Antimatter, Dark Matter, Trento, 1998 (unpublished).

[15] AMS Collaboration, “Alpha Magnetic Spectrometer for Extraterrestrial Study of Antimatter, Matter and Missing Matter on the International Space Station Alpha," proposal, 1994 (unpublished); S. Ahlen et al., Nucl. Instrum. Methods Phys. Res. A 350, 351 (1994).

[16] R. Bellotti et al., "PAMELA: an Antiproton, Positron Experiment on a Polar Orbit Satellite,' Report No. DFF/240, 1995; O. Adriani et al., in Proceedings of the 24th International Conference of Cosmic Rays [3].

[17] For a review on these future measurements see, for instance, P. Spillantini, in TAUP97, Proceedings of TAUP97, edited by A. Bottino, A. Di Credico, and P. Monacelli [Nucl. Phys. B (Proc. Suppl.) (in press)].

[18] T.K. Gaisser and R.K. Schaefer, Astrophys. J. 394, 174 (1992), and references therein.

[19] IMAX Collaboration, W. Menn et al., in Proceedings of the 25th International Conference of Cosmic Rays [1].

[20] WiZard/CAPRICE Collaboration, M. Boezio et al., Report No. INFN/AE-98/06 (unpublished).

[21] J.S. Perko, Astron. Astrophys. 184, 119 (1987).

[22] J.S. Perko, Astrophys. J. 397, 153 (1992).

[23] C. Rastoin et al., Astron. Astrophys. 307, 981 (1996). 
[24] P. Papini, C. Grimani, and S.A. Stephens, Nuovo Cimento C 19, 367 (1996).

[25] L.C. Tan and L.K. Ng, Phys. Rev. D 26, 1179 (1982).

[26] A recent analysis by R.P. Olling and M.R. Merrifield, astro-ph/9710224, and R.P. Olling (private communication), points to a more stringent bound on the Milky Way flattening $(f \gtrsim 0.5)$ and to an Earth's galactocentric distance $\left(r_{\odot}=7.0\right.$ $\pm 1.0 \mathrm{kpc})$ smaller than the usual one $\left(r_{\odot}=8.0 \pm 1.0 \mathrm{kpc}\right)$.

[27] V. Berezinsky, A. Bottino, J. Ellis, G. Mignola, and S. Scopel, Astropart. Phys. 5, 333 (1996).

[28] A. Bottino, V. de Alfaro, N. Fornengo, G. Mignola, and M. Pignone, Astropart. Phys. 2, 67 (1994).

[29] J. Binney and S. Tremaine, Galactic Dynamics (Princeton University Press, Princeton, NJ, 1987).

[30] E.I. Gates, G. Gyuk, and M.S. Turner, Astrophys. J. Lett. 449, L123 (1995).

[31] H.P. Nilles, Phys. Rep. 110, 1 (1984); H.E. Haber and G.L. Kane, ibid. 117, 75 (1985); R. Barbieri, Riv. Nuovo Cimento 11, 1 (1988).

[32] L. Bergström and P. Ullio, Nucl. Phys. B504, 27 (1997).

[33] T. Sjöstrand, Comput. Phys. Commun. 39, 347 (1986); 43, 367 (1987); Report No. CERN-TH 6488/92.

[34] ALEPH Collaboration, P. Dornan, ALEPH Collaboration, P. Charpentier, Presentations at the LEPC Conference, 1997 (unpublished).

[35] CLEO Collaboration, M.S. Alam et al., Phys. Rev. Lett. 74, 2885 (1995).

[36] W.R. Webber, M.A. Lee, and M. Gupta, Astrophys. J. 390, 96 (1992).

[37] A.G. Lyne, R.N. Manchester, and J.H. Taylor, Mon. Not. R. Astron. Soc. 213, 613 (1985).

[38] L.C. Tan and L.K. Ng, J. Phys. G 9, 227 (1983).

[39] Seo et al., in Proceedings of the 23rd International Conference of Cosmic Rays, Calgary, 1993, edited by R. B. Hicks, D. A. Leahy, and D. Venkatesan (World Scientific, River Edge, NJ, 1994).

[40] Ficenec et al., in Proceedings of the 23rd International Conference of Cosmic Rays [39].

[41] P. Ferrando, Saclay Report No. SAP 1993/16; Proceedings of the 23rd International Conference of Cosmic Rays, Calgary, 1993, Rapporteur talks and highlight papers, 1994, edited by J. Wdowczyk, B. V. Sreekantan, and H. Moraal (World Scientific, River Edge, NJ, 1994), p. 279.

[42] E. Eisenhandler et al., Nucl. Phys. B113, 1 (1976).
[43] P. Chardonnet, G. Mignola, P. Salati, and R. Taillet, Phys. Lett. B 384, 161 (1996).

[44] G. Mignola, in Proceedings of the First International Workshop on The Identification of Dark Matter, Sheffield, 1996, astro-ph/9611138.

[45] See, for instance, Ref. [5] for a brief overview of this matter.

[46] These data are consistent with the results of two other recent experiments $[4,6]$.

[47] Since the BESS95 data have asymmetric error bars, we define a generalized $\chi^{2}$ as

$$
\begin{aligned}
\chi^{2}= & \sum_{i}\left\{\frac{\left[\Phi^{\mathrm{th}}\left(T_{i}\right)-\Phi_{i}^{\mathrm{exp}}\right]^{2}}{\left(\sigma_{i}^{+}\right)^{2}} \theta\left[\Phi^{\mathrm{th}}\left(T_{i}\right)-\Phi_{i}^{\exp }\right]\right. \\
& \left.+\frac{\left[\Phi^{\mathrm{th}}\left(T_{i}\right)-\Phi_{i}^{\exp }\right]^{2}}{\left(\sigma_{i}^{-}\right)^{2}} \theta\left[\Phi_{i}^{\exp }-\Phi^{\mathrm{th}}\left(T_{i}\right)\right]\right\},
\end{aligned}
$$

where $\sigma_{i}^{+}\left(\sigma_{i}^{-}\right)$is the positive (negative) uncertainty in the $i$ th energy bin and $\theta$ is the Heaviside step function. In the case of symmetric uncertainties $\left(\sigma_{i}^{+}=\sigma_{i}^{-} \equiv \sigma_{i}\right)$ this formula gives the standard definition of $\chi^{2}$.

[48] H. Bengtsson, P. Salati, and J. Silk, Nucl. Phys. B346, 129 (1990); J. Silk and A. Stebbins, Astrophys. J. 411, 439 (1993); V. Berezinsky, A. Bottino, and G. Mignola, Phys. Lett. B 391, 355 (1997); T. Mitsui, K. Maki, and S. Orito, ibid. 389, 169 (1996); L. Bergström, J. Edsjö, and P. Ullio, astro-ph/9804050.

[49] A. Bottino, F. Donato, G. Mignola, S. Scopel, P. Belli, and A. Inchicchitti, Phys. Lett. B 402, 113 (1997), and references therein.

[50] For an up-to-date overview of direct searches for particle dark matter see, for instance, D.O. Caldwell, in Proceedings of TAUP97 [17].

[51] D. Reusser et al., Phys. Lett. B 255, 143 (1991); R. Bernabei et al., ibid. 389, 757 (1996).

[52] R. Bernabei et al., astro-ph/9710290; in Proceedings of TAUP97 [17]; Phys. Lett. B 424, 195 (1998).

[53] A. Bottino, F. Donato, N. Fornengo, and S. Scopel, Phys. Lett. B 423, 109 (1998); Report No. DFTT 61/97, hep-ph/9710295.

[54] "'Physics at LEP2,' edited by G. Altarelli, T. Sjöstrand, and F. Zwirner, CERN Report No. 96-01, 1996; F. Richard, Report No. LAL 98-04, 1998 (unpublished).

[55] ' Report of the TeV-2000 Study Group,'” edited by D. Amidei and R. Brock, Report No. FERMILAB-PUB-96/082, 1996.

[56] V. Berezinsky, A. Bottino, J. Ellis, N. Fornengo, G. Mignola, and S. Scopel, Astropart. Phys. 5, 1 (1996).

[57] R. Battiston (private communication). 